\title{
Rotational Analysis of the Red Electronic Emission Spectrum of Molybdenum Nitride (MoN)
}

\author{
R. C. CARlson, ${ }^{1}$ J. K. Bates, ${ }^{2}$ AND T. M. DunN \\ The Department of Chemistry, University of Michigan, Ann Arbor, Michigan 48109
}

\begin{abstract}
A system of emission bands in the red region of the optical spectrum has been identified as due to the species MoN. The system was generated by the microwave $(2450 \mathrm{MHz})$ excitation of a flowing mixture of $\mathrm{MoCl}_{5}$ and molecular nitrogen in a stream of helium but is also observed in a DC arc in air between molybdenum electrodes. One of the $Q$-form branches has previously been assumed to be an atomic line of Mo $\mathrm{l}$. The system has been assigned as the 0 , 0 band of a ${ }^{4} \Pi(a) \rightarrow X^{4} \Sigma^{-}(a)$ transition, with a large zero-field splitting of the ground ${ }^{4} \Sigma^{-}$ term $\left(\sim 86 \mathrm{~cm}^{-1}\right)$. A preliminary search has been made to detect the presence of MoN in Mtype stars. (1) 1985 Academic Press, Inc.
\end{abstract}

\section{INTRODUCTION}

In contrast to the transition metal oxide emission spectra there are, so far, relatively few transition metal nitride systems either known or analyzed. The species identified in the gas phase are $\operatorname{TiN}(1,2), \mathrm{ZrN}(3), \mathrm{NbN}(4,5), \operatorname{MoN}(6,7), \mathrm{VN}$ $(8)$, TaN (9), and ReN (10), while matrix isolation studies have been carried out on $\mathrm{TaN}(11), \mathrm{ZrN}(12), \mathrm{MoN}(13,14)$, and $\mathrm{NbN}(15)$. In fact, the first authenticated example of a transition metal nitride system was that of $\mathrm{MoN}$, which was recognized by Howard and Conway (6) in 1965 from the results of an ${ }^{15} \mathrm{~N}$ isotopic experiment. The systems were not, however, rotationally analyzed and, when generated by an arc between molybdenum electrodes in nitrogen, the lines are badly broadened from Doppler and multiple molybdenum isotope sources. [Natural abundance molybdenum has seven isotopes with mass numbers and abundances of $92(15.84 \%), 94$ (9.04\%), $95(15.72 \%), 96(16.53 \%), 97(9.46 \%), 98(23.78 \%)$ and $100(9.63 \%)$.]

In addition to the rarity of nitride systems, there are few examples (16-23) of transitions between terms with quartet spin states, particularly where there is a large zero-field splitting of a ${ }^{4} \Sigma^{-}$state (24) requiring its classification as Hund's case (a) $(25,26)$. The present example involves both of these circumstances.

Finally, it is worth noting that molybdenum appears to be unique among the transition metals in its generation of a nitride in an arc in air. The ramifications are that there may be indication of $\mathrm{MoN}$ in the cool (M and $\mathrm{S}$ type) stars, and even though this phenomenon cannot be directly connected with the role of molybdenum in nitrogen fixation by legumes it may, nonetheless, be indirectly related to it.

\footnotetext{
' Department of Chemistry, University of California, and Materials and Molecular Research Division, Lawrence Berkeley Laboratory, Berkeley, Calif. 94720.

${ }^{2}$ Chemical Technology Division, Argonne National Laboratory, Argonne, Ill. 60439.
} 


\section{EXPERIMENTAL DETAILS}

The spectra of all systems were obtained from the reaction of isotopically enriched $(\sim 85 \%){ }^{94} \mathrm{MoCl}_{5}$ and active nitrogen (and also $\left.{ }^{15} \mathrm{~N}_{2}\right)$ in a microwave discharge $(\sim 100 \mathrm{~W})$ using helium as the pumping gas. The spectra were photographed on $103 \mathrm{aD}, 103 \mathrm{aF}$, II $\mathrm{aD}$, and II aF plates and films on a $1.5-\mathrm{m}$ Bausch and Lomb spectrograph for low resolution $(\sim 15 \AA / \mathrm{mm}$ in the first order) and in the 9th and 10th orders of a Jarrell-Ash 3.4-m Ebert spectrograph. The resolution is $\sim 500,000$ in the 10th order of the latter instrument and the reciprocal dispersion is $\sim 3 \AA / \mathrm{cm}$.

Standard wavelength calibrations used were neon, thorium (in a microwave excited discharge), and a hollow cathode $\mathrm{Fe}$ lamp. The standard lines were first fitted to a quadratic equation, any badly fitting lines being discardcd or checked for errors. The lines were finally fitted to a fourth-order polynomial with a standard deviation of not greater than $0.001 \AA$, any suspect lines again being discarded. The wavelengths of the unblended spectral lines were interpolated from this polynomial, having been measured to an accuracy of $\sim 1 \mu \mathrm{m}$, and were converted to vacuum wavelengths and $\mathrm{cm}^{-1}$. Final errors in the line positions have been estimated as $\pm 0.003 \mathrm{~cm}^{-1}$.

The line frequencies are listed in Tables Ia, b, c, and $d$ in $\mathrm{cm}^{-1}$ (vacuum corrected).

\section{APPEARANCE OF SYSTEM}

The emission from MoN is quite intense under these experimental conditions and the discharge has a distinct reddish aureole. Under low resolution (Fig. 1) the region between 5990 and $6350 \AA$ is dominated by molecular bands. The carrier was confirmed from the ${ }^{15} \mathrm{~N}$ spectra as $\mathrm{MoN}$ and the bandheads closely match chose given by Howard and Conway (6). An exception is the intense line-like feature at $6123 \AA$ which shows a negligible isotope shift and is identified in the MIT tables as an atomic line of Mo. As is shown below, this feature is really a complex combination of $Q$-form bandheads.

Under high resolution, the band system clearly represents one of the most spectacular examples of a high-spin multiplicity transition. Aside from some weak sequence bands there are very few interfering lines of any kind, there are no obvious perturbations, and the intensity is sufficient to allow the lines to be recorded up to $J \sim 60$.

The high-resolution photographs of the systems are given in Figs. 2a, b, c, and d. It will be immediately observed that the 6305 - and $5996-\AA$ systems are very similar in appearance, with spacings between lines at low values of $J$ of $0 B$ and $\pm 2 B$. The $6245-$ and $6123-\AA$ systems are also very similar, with low $J$ spacings of $0 B, \pm 2 B$, and $\pm 4 B$. (The wavelengths indicated correspond to the subband origins, $\nu_{0}$.) The former pair are typical of many previously known subbands in that they consist of doubled $P, Q$, and $R$ branches with a conventional $J$ dependence and degradation. The latter pair are quite distinct, however, in their unusual $Q$-form, $O$, and $S$-form features in addition to the more usual $P$ and $R$ branches. 
TABLE Ia

Line Frequencies for the ${ }^{4} \mathrm{I}_{-1 / 2} \rightarrow{ }^{4} \Sigma_{3 / 2}$ Transition of $\mathrm{MoN}$

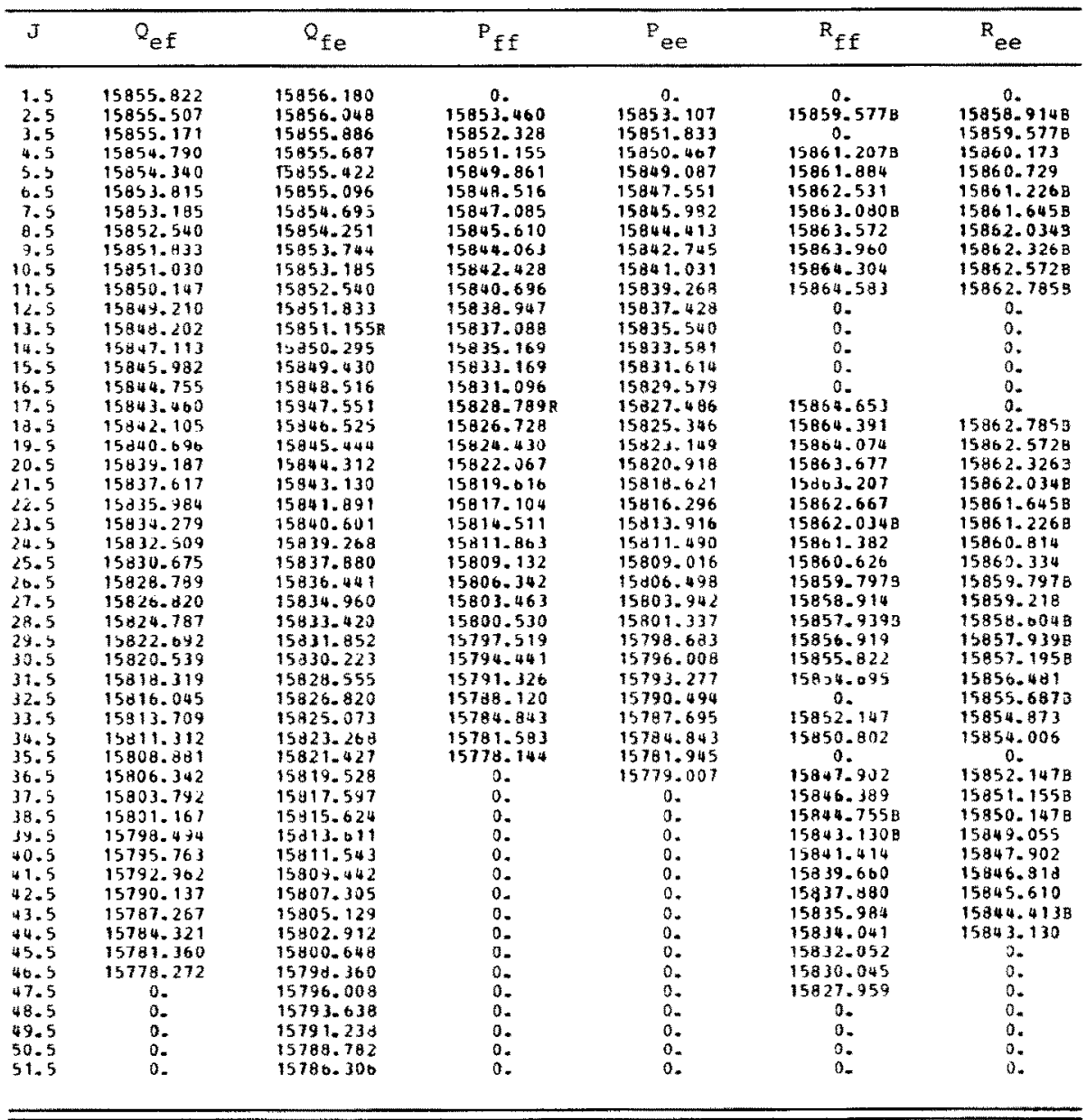

$B=$ BLENDED LINE

$R=$ REJECTED LINE

\section{RESULTS AND ANALYSIS}

The four subbands described above have been assigned as the ${ }^{4} \Pi_{-1 / 2}(a) \rightarrow{ }^{4} \Sigma_{3 / 2}^{-}(a)$ $(6305 \AA),{ }^{4} \Pi_{1 / 2}(a) \rightarrow{ }^{4} \Sigma_{1 / 2}^{-}(a)(6245 \AA),{ }^{4} \Pi_{3 / 2}(a) \rightarrow{ }^{4} \Sigma_{1 / 2}^{-}(a)(6123 \AA)$, and ${ }^{4} \Pi_{5 / 2}(a) \rightarrow{ }^{4} \Sigma_{3 / 2}^{-}(a)(5996 \AA)$ components of the $(0,0)$ band of a ${ }^{4} \Pi(a) \rightarrow{ }^{4} \Sigma^{-}(a)$ transition. This assignment was based, first, upon the general appearance of the bands, as cited above; second, by a subband by subband analysis; and, third, upon a concerted analysis of the system. The gross appearance of the system can be derived from the energy level diagram given in Fig. 1 and it reflects the case (a) selection rules $\Delta \Sigma=0$ and $\Delta \Omega=0, \pm 1$. It will be noted that there is a very small 
TABLE Ib

Line Frequencies for the ${ }^{4} \Pi_{1 / 2} \rightarrow{ }^{4} \Sigma_{1 / 2}^{-}$Transition of MoN

\begin{tabular}{|c|c|c|c|c|c|c|}
\hline $\mathrm{J}$ & $Q_{\text {ef }}$ & $Q_{\text {fe }}$ & $P_{f \pm}$ & $P_{\text {ee }}$ & $R_{f \neq}$ & $R_{\text {ee }}$ \\
\hline $\begin{array}{l}1.5 \\
2.5 \\
3.5 \\
4.5 \\
5.5 \\
6.5 \\
7.5 \\
6.5 \\
9.5 \\
10.5 \\
11.5 \\
12.5 \\
13.5 \\
14.5 \\
15.5 \\
16.5 \\
17.5 \\
18.5 \\
19.5 \\
20.5 \\
21.5 \\
22.5 \\
23.5 \\
24.5 \\
25.5 \\
26.5 \\
27.5 \\
28.5 \\
24.5 \\
30.5 \\
31.5 \\
32.5 \\
33.5 \\
34.5 \\
35.5 \\
36.5 \\
37.5 \\
38.5 \\
39.5 \\
40.5 \\
41.5 \\
42.5 \\
43.5 \\
44.5 \\
45.5 \\
46.5 \\
47.5 \\
48.5 \\
49.5 \\
50.5 \\
51.5 \\
52.5 \\
53.5 \\
54.5 \\
55.5 \\
56.5 \\
57.5 \\
59.5 \\
60.5\end{array}$ & $\begin{array}{c}16004.792 \\
16003.898 \\
16003.024 \\
16002.1373 \\
15001.269 \\
16000.406 \\
15999.559 \\
15998.7108 \\
15997.870 \mathrm{~B} \\
15997.046 \\
15996.234 \\
15995.426 \\
15494.628 \\
15993.844 \\
15993.070 \\
15992.305 \\
15991.549 \\
15990.913 \\
15990.079 \\
15989.352 \\
15983.649 \\
15987.944 \\
15987.252 \\
15986.567 \\
15985.896 \\
15985.228 \\
15984.565 \\
15983.9138 \\
15983.261 \\
15982.017 \\
15981.974 \mathrm{~B} \\
15981.346 \\
15990.103 \\
15920.071 \\
15979.427 \\
15978.804 \\
15978.160 \\
15977.522 \\
15976.871 \\
0 . \\
15975.554 \\
15974.903 \\
15974.228 \\
0 . \\
0 . \\
0 . \\
0 . \\
0 . \\
0 . \\
0 . \\
3 . \\
0 . \\
0 . \\
0 . \\
0 . \\
0 . \\
0 . \\
0 . \\
0 . \\
0 . \\
0 . \\
0 . \\
0 .\end{array}$ & $\begin{array}{c}0 . \\
0 . \\
16010.253 \\
16011.188 \\
16012.107 \\
16013.042 \\
16013.975 \\
16014.912 \\
16015.846 \\
16016.781 \\
16017.714 \\
16018.649 \\
16019.582 \\
16020.514 \\
16021.445 \\
16022.376 \\
16023.293 \\
16024.212 \\
16025.122 \\
16026.031 \\
16026.928 \\
16027.321 \\
16028.709 \\
16029.586 \\
16030.455 \\
16031.311 \\
16032.159 \\
16032.997 \\
16033.819 \\
16034.635 \\
16035.4218 \\
16036.218 \\
16036.990 \\
16037.749 \\
16038.486 \\
16039.213 \\
16039.926 \\
16040.017 \\
16041.268 \\
16041.942 \\
16042.584 \\
16043.201 \\
16043.799 \\
16044.379 \\
16044.937 \\
16045.476 \\
16045.9953 \\
16046.482 \\
16046.956 \\
16047.405 \\
16047.833 \\
16048.1421 \\
16048.613 \\
16048.976 \\
16049.299 \\
16049.511 \\
16049.888 \\
16050.138 \\
16050.371 \\
16050.583\end{array}$ & 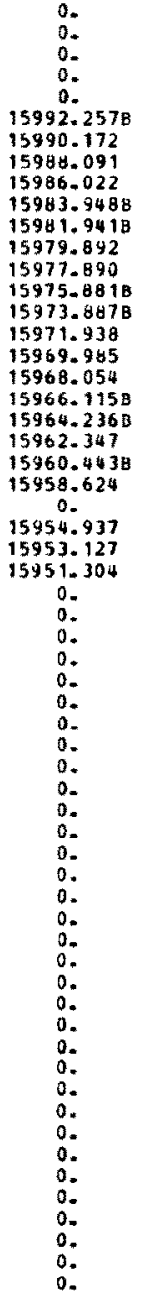 & $\begin{array}{c}0 . \\
0 . \\
0 . \\
0 . \\
0 . \\
0 . \\
0 . \\
0 . \\
0 . \\
0 . \\
0 . \\
0 . \\
0 . \\
0 . \\
0 . \\
0 . \\
0 . \\
0 . \\
0 . \\
0 . \\
0 . \\
0 . \\
0 . \\
0 . \\
0 . \\
0 . \\
0 . \\
0 . \\
0 . \\
0 . \\
0 . \\
0 . \\
0 . \\
0 . \\
0 . \\
16008.445 \\
16008.243 \\
16008.020 \\
16007.784 \\
0 . \\
16007.256 \\
16006.933 \\
16006.020 \\
0 . \\
16005.930 \\
16005.538 \\
16005.127 \\
16004.685 \\
16004.225 \\
16003.738 \\
10003.233 \\
16002.699 \\
16002.1378 \\
16001.546 \\
16000.941 \\
0 . \\
0 . \\
0 . \\
0 . \\
0 . \\
0 .\end{array}$ & $\begin{array}{c}0 . \\
0 . \\
0 . \\
0 . \\
0 . \\
0 . \\
0 . \\
0 . \\
0 . \\
0 . \\
0 . \\
0 . \\
0 . \\
0 . \\
0 . \\
16006.423 \\
16006.515 \\
16006.628 \\
16006.742 \\
16006.872 \\
16007.015 \\
16007.177 \\
16007.353 \\
0 . \\
0 . \\
0 . \\
16008.170 \\
0 . \\
0 . \\
0 . \\
0 . \\
0 . \\
16009.658 \\
16009.927 \\
16010.215 \\
16010.471 \\
16010.749 \\
16011.017 \\
16011.283 \\
16011.5528 \\
160: 1.820 \\
0 . \\
0 . \\
0 . \\
0 . \\
0 . \\
0 . \\
0 . \\
0 . \\
0 . \\
0 . \\
0 . \\
0 . \\
0 . . \\
0.0 \\
0 . \\
0 . \\
0 . \\
0 . \\
0 . \\
0 .\end{array}$ & $\begin{array}{l}16011.5528 \\
16013.6928 \\
16015.8468 \\
16018.063 \\
16020.242 \\
10022.370 \mathrm{~B} \\
16024.585 \\
16026.755 \\
16028.920 \\
16031.082 \\
16033.240 \\
16035.2411 \\
16037.534 \\
10039.670 \\
16041.809 \\
16043.925 \\
16046.0289 \\
16048.2358 \\
16050.239 \\
16052.317 \\
16054.389 \\
16056.448 \\
16058.491 \\
16060.525 \\
16062.546 \\
16064.542 \\
16066.532 \\
16068.504 \\
16070.458 \\
16072.399 \\
16074.320 \\
16076.222 \\
16078.112 \\
16079.971 \\
16081.917 \\
16081.643 \\
16095.442 \\
16087.226 \\
16088.995 \\
16090.730 \\
16092.451 \\
16094.145 \\
16095.814 \\
16097.465 \\
16099.090 \\
10100.687 \\
16102.261 \\
16103.8114 \\
16105.336 \\
16106.929 \\
16108.301 \\
16109.752 \\
16111.169 \\
161112.560 \\
16113.927 \\
16115.259 \\
16116.578 \\
16117.826 \\
16119.097 \\
16120.325\end{array}$ \\
\hline
\end{tabular}

interval for the ${ }^{4} \Pi_{1 / 2} \rightarrow{ }^{4} \Pi_{-1 / 2}$ spacing $\left(\sim 65 \mathrm{~cm}^{-1}\right)$ but that the spacings for the other ${ }^{4} \mathrm{II}$ components are as would be expected for a second-row transition element with a one-electron spin-orbit coupling constant $\sim 300 \mathrm{~cm}^{-1}$. Perhaps the most surprising feature is the very large zero-field splitting of the ${ }^{4} \Sigma^{-}$state, $\sim 86 \mathrm{~cm}^{-1}$, thereby clearly classifying it as an example of Hund's case (a) coupling. The very 
TABLE Ic

Line Frequencies for the ${ }^{4} \Pi_{3 / 2} \rightarrow{ }^{4} \Sigma_{1 / 2}^{-}$Transition of MoN

\begin{tabular}{|c|c|c|c|c|c|c|}
\hline $\mathrm{J}$ & $Q_{e f}$ & $Q_{\text {fe }}$ & $P_{\text {ff }}$ & $P_{\text {ee }}$ & $R_{f f}$ & $\mathrm{R}_{\text {ee }}$ \\
\hline $\begin{array}{l}1.5 \\
1.5 \\
2.5 \\
3.5 \\
4.5 \\
5.5 \\
6.5 \\
7.5 \\
8.5 \\
9.5 \\
10.5 \\
11.5 \\
12.5 \\
13.5 \\
14.5 \\
15.5 \\
16.5 \\
17.5 \\
18.5 \\
19.5 \\
20.5 \\
21.5 \\
22.5 \\
23.5 \\
24.5 \\
25.5 \\
26.5 \\
27.5 \\
28.5 \\
23.5 \\
30.5 \\
31.5 \\
32.5 \\
33.5 \\
34.5 \\
35.5 \\
36.5 \\
37.5 \\
38.5 \\
39.5 \\
40.5 \\
41.5 \\
42.5 \\
43.5 \\
44.5 \\
45.5 \\
46.5 \\
47.5 \\
48.5 \\
49.5 \\
50.5 \\
51.5 \\
52.5 \\
53.5 \\
54.5 \\
55.5 \\
56.5 \\
57.5 \\
58.5 \\
59.5 \\
60.5\end{array}$ & $\begin{array}{c}0 . \\
0 . \\
16320.900 \\
16319.905 \\
16318.862 \\
16317.826 \\
16316.787 \\
16315.747 \\
16314.7078 \\
16313.680 \\
16312.644 \\
16311.617 \\
16310.592 \\
16309.572 \\
16308.552 \\
16307.530 \\
16306.534 \\
16305.533 \\
16304.530 \\
16303.542 \\
16302.557 \\
16301.579 \\
16300.601 \\
16299.629 \\
16298.663 \\
16297.698 \\
16296.743 \\
16295.736 \\
16294.4318 \\
16293.891 \\
16292.421 \\
16291.993 \\
16291.045 \\
16290.096 \\
16289.147 \\
16288.144 \\
16287.246 \\
16286.289 \\
16285.336 \\
16284.364 \\
16283.402 \\
16282.422 \\
0.4 . \\
16230.455 \\
16279.456 \\
16278.443 \\
16277.434 \\
16276.415 \\
16275.373 \\
16274.325 \\
16273.281 \\
0.39 \\
16271.1117 \\
16270.020 \\
16268.922 \\
16267.786 \\
16266.651 \\
16265.490 \\
16264.325 \\
0 .\end{array}$ & 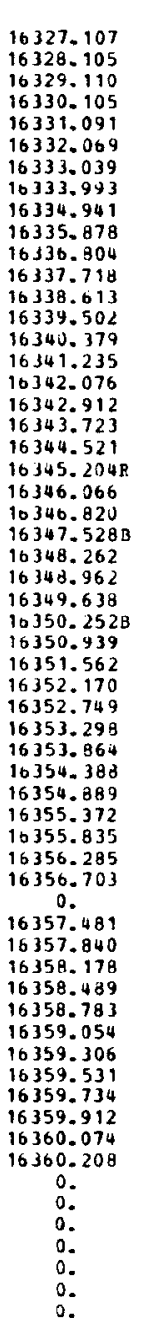 & $\begin{array}{c}0 . \\
0 . \\
0 . \\
0 . \\
16313.309 \\
16311.268 \\
16309.214 \\
16307.160 \\
16305.106 \\
16303.056 \\
16301.027 \\
16298.979 \\
16296.949 \\
16298.9238 \\
0 . \\
16240.872 \\
16288.830 \\
16286.935 \\
0.9 \\
16282.820 \\
16280.830 \\
16278.839 \\
16276.855 \\
16274.876 \\
16272.898 \\
16270.929 \\
16268.955 \\
16266.999 \\
16265.021 \\
16263.087 \\
16263.119 \\
16259.163 \\
16257.210 \\
16255.255 \\
16253.292 \\
16251.348 \\
16249.380 \\
16247.416 \\
16245.447 \\
16243.471 \\
16241.490 \\
16239.514 \\
16204.366 \\
16202.175 \\
16237.531 \\
16235.5198 \\
16233.532 \\
16231.522 \\
16229.498 \\
16227.475 \\
16225.424 \\
16223.384 \\
16221.321 \\
16219.238 \\
16217.164 \\
16215.068 \\
16212.959 \\
16210.812 \\
16208.686 \\
169\end{array}$ & $\begin{array}{c}0 . \\
0 . \\
0 . \\
0 . \\
0 . \\
0 . \\
0 . \\
0 . \\
0 . \\
0 . \\
0 . \\
0 . \\
16324.976 \\
16324.862 \\
16324.729 \\
16324.564 \\
16324.418 \\
16324.242 \\
16324.046 \\
16323.922 \\
16323.610 \\
16323.371 \\
16323.119 \\
16322.845 \\
16322.553 \\
16322.242 \\
16321.927 \\
16321.5169 \\
16321.228 \\
16320.843 \\
16320.450 \\
10320.032 \\
16319.600 \\
16319.139 \\
16318.668 \\
16318.175 \\
16317.662 \\
16317.134 \\
16316.580 \\
16316.004 \\
16315.412 \\
16314.7908 \\
16314.166 \\
16313.510 \\
16312.831 \\
16312.142 \\
16311.4168 \\
16310.678 B \\
16309.916 \\
16309.134 \\
16308.337 \\
0.0 \\
16306.651 \\
16305.782 \\
16304.895 \\
16303.980 \\
0.9 \\
16302.081 \\
16301.0858 \\
16300.098\end{array}$ & $\begin{array}{l}0 . \\
0 . \\
0 . \\
0 . \\
0 . \\
0 . \\
0 . \\
0 . \\
0 . \\
0 . \\
0 . \\
0 . \\
0 . \\
0 . \\
0 . \\
0 . \\
0 . \\
0 . \\
0 . \\
0 . \\
0 . \\
0 . \\
0 . \\
0 . \\
0 . \\
0 . \\
0 . \\
0 . \\
0 . \\
0 . \\
0 . \\
0 . \\
0 . \\
0 . \\
0 . \\
0 . \\
0 . \\
0 . \\
0 . \\
0 . \\
0 . \\
0 . \\
0 . \\
0 . \\
0 . \\
0 . \\
0 . \\
0 . \\
0 . \\
0 . \\
0 . \\
0 . \\
0 . \\
0 . \\
0 . \\
0 . \\
0 . \\
0 . \\
0 . \\
0 . \\
.0\end{array}$ & $\begin{array}{r}16327.599 \\
16329.621 \\
16331.641 \\
16333.651 \\
16335.660 \\
0 . \\
16339.647 \\
16341.624 \\
16343.599 \\
16345.552 \\
0 . \\
16349.436 \\
16351.355 \\
0 . \\
16355.164 \\
0.9 \\
16358.905 \\
16360.764 \\
16362.611 \\
16364.429 \\
16366.237 \\
16368.022 \\
16369.904 \\
16371.559 \\
16373.299 \\
16375.023 \\
16376.729 \\
16378.415 \\
16390.085 \\
16391.737 \\
16383.369 \\
16384.980 \\
10386.575 \\
15388.145 \\
16389.709 \\
16391.236 \\
16392.749 \\
16394.233 \\
16395.706 \\
16397.151 \\
16398.587 \\
16399.991 \\
16401.373 \\
16402.744 \\
16404.083 \\
16405.401 \\
16406.701 \\
16407.973 \\
16409.230 \\
16410.457 \\
16411.663 \\
16412.847 \\
16414.004 \\
16415.138 \\
16416.253 \\
16417.348 \\
16418.412 \\
16419.451 \\
16420.473 \\
16421.473 \\
16422.448\end{array}$ \\
\hline
\end{tabular}

unusual appearance of the $Q$-form (line-like) features in the 6123- and $6245-\AA$ subbands is the result of the combination of a large $\Omega$-type splitting of the ${ }^{4} \Sigma_{1 / 2}^{-}$ state upon which both terminate and the $\Lambda$-doubling of the ${ }^{4} \Pi$ substates from which the transitions arise.

The analysis of the spectrum has been performed in three successive stages: the subband by subband analysis $\left({ }^{4} \Pi_{\Omega} \rightarrow{ }^{4} \Sigma_{\Omega}^{-}\right)$, the intermediate analysis $\left[{ }^{4} \Pi_{\Omega} \rightarrow{ }^{4} \Sigma^{-}(a)\right]$, 
TABLE Id

Line Frequencies for the ${ }^{4} \Pi_{5 / 2} \rightarrow{ }^{4} \Sigma_{3 / 2}^{-}$Transition of MoN

\begin{tabular}{|c|c|c|c|c|c|c|}
\hline$J$ & $Q_{e f}$ & $Q_{\mathrm{fe}}$ & $P_{f f}$ & $P_{\text {ee }}$ & $R_{f f}$ & $\mathrm{R}_{\text {ee }}$ \\
\hline $\begin{array}{l}1.5 \\
2.5 \\
3.5 \\
1.5 \\
5.5 \\
6.5 \\
7.5 \\
8.5 \\
9.5 \\
10.5 \\
11.5 \\
12.5 \\
13.5 \\
14.5 \\
13.5 \\
16.5 \\
17.5 \\
18.5 \\
19.5 \\
20.5 \\
21.5 \\
22.5 \\
23.5 \\
24.5 \\
25.5 \\
26.5 \\
27.5 \\
28.5 \\
29.5 \\
30.5 \\
31.5 \\
32.5 \\
33.5 \\
34.5 \\
35.5 \\
36.5 \\
37.5 \\
38.5 \\
34.5 \\
40.5 \\
41.5 \\
42.5 \\
59.5 \\
61.5 \\
43.5 \\
44.5 \\
45.5 \\
46.5 \\
47.5 \\
48.5 \\
49.5 \\
50.5 \\
51.5 \\
52.5 \\
53.5 \\
54.5 \\
56.5 \\
57\end{array}$ & $\begin{array}{l}0 . \\
16672.8308 \\
16572.6848 \\
16672.4948 \\
16672.2748 \\
16671.987 \\
16671.558 \\
16671.288 \\
16670.870 \\
16670.411 \\
16669.897 \\
16669.340 \\
16668.742 \\
16668.088 \\
16667.380 \\
16666.621 \\
16665.817 \\
16604.956 \\
16664.056 \\
16603.104 \\
16662.097 \\
16661.003 \\
16653.941 \\
16658.786 \\
16657.378 \\
16656.328 \\
16655.030 \\
16653.673 \\
16652.273 \\
16650.829 \\
16649.348 \\
16047.802 \\
16646.229 \\
16644.595 \\
16642.919 \\
16541.206 \\
16039.449 \\
16637.548 \\
16635.305 \\
16633.927 \\
16632.007 \\
16630.053 \\
16628.059 \\
16626.018 \\
16623.953 \\
16621.855 \\
16619.686 \\
16617.519 \\
16615.317 \\
16613.074 \\
16610.796 \\
16608.483 \\
16606.145 \\
16603.769 \\
16601.364 \\
16598.928 \\
16596.457 \\
16593.952 \\
16591.450 \\
16589.906 \\
16586.3228\end{array}$ & 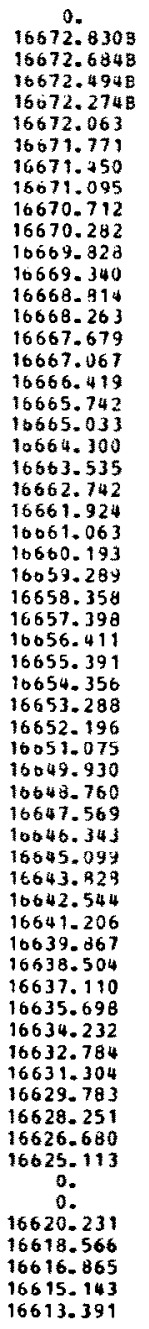 & $\begin{array}{c}0 . \\
0 . \\
0 . \\
0 . \\
0 . \\
16665.409 \\
0 . \\
0 . \\
16661.256 \\
16659.798 \\
16658.282 \\
16656.713 \\
0 . \\
16653.417 \\
16651.700 \\
16649.930 \\
16648.122 \\
0 . \\
16644.326 \\
16642.386 \\
16640.357 \\
16638.302 \\
16636.190 \\
16634.020 \\
16631.807 \\
16629.4928 \\
16627.240 \\
16624.885 \\
16622.469 \\
16620.023 \\
16617.519 \\
16614.968 \\
16612.390 \\
16609.757 \\
16507.087 \\
16604.372 \\
16601.592 \\
16594.804 \\
16595.960 \\
16543.071 \\
16590.155 \\
16587.188 \\
16584.196 \\
16581.157 \\
0 . \\
16574.995 \\
16571.854 \\
16568.669 \\
16565.455 \\
16562.219 \\
16558.947 \\
16555.626 \\
16552.310 \\
16548.935 \\
16545.536 \\
0 . \\
16538.641 \\
0 . \\
16531.647 \\
16528.106 \\
0 .\end{array}$ & $\begin{array}{c}0 . \\
0 . \\
0 . \\
0 . \\
0 . \\
16655.485 \\
0 . \\
16662.839 \\
16661.480 \\
16060.096 \\
16658.649 \\
16657.189 \\
16655.685 \\
16654.157 \\
16652.584 \\
16650.993 \\
16649.348 \\
16647.723 \\
16646.026 \\
16644.126 \\
16642.544 \\
16640.786 \\
16638.980 \\
16037.156 \\
16635.310 \\
16633.419 \\
16631.503 \\
16629.412 \\
16627.598 \\
16625.598 \\
16623.584 \\
16621.540 \\
16519.464 \\
16617.356 \\
16615.240 \\
16613.074 \\
16510.907 \\
16608.113 \\
16606.502 \\
16604.245 \\
16601.970 \\
16599.672 \\
16597.359 \\
16595.011 \\
16592.630 \\
16590.247 \\
16587.858 \\
16585.393 \\
16582.934 \\
16580.451 \\
0 . \\
16575.405 \\
16572.859 \\
16570.291 \\
16567.672 \\
16565.064 \\
16562.427 \\
16559.750 \\
16557.068 \\
16554.354 \\
0 .\end{array}$ & 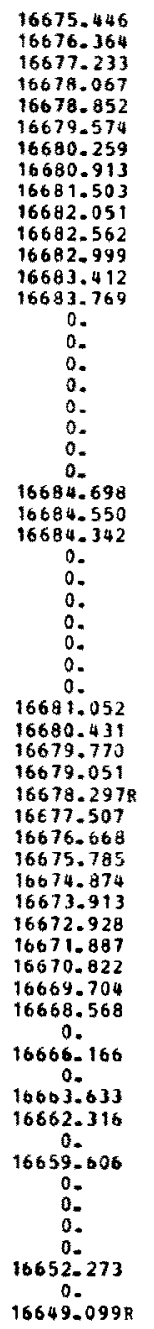 & $\begin{array}{c}0 . \\
0 . \\
0 . \\
0 . \\
0 . \\
16679.655 \\
16680.365 \\
16681.052 \\
16681.716 \\
16682.336 \\
16682.925 \\
16683.478 \\
16684.023 \\
0 . \\
16684.953 \\
16685.367 \\
16685.754 \\
16886.130 \\
16696.477 \\
16686.777 \\
16687.049 \\
16687.299 \\
16687.516 \\
16687.703 \\
16687.855 \\
0 . \\
0 . \\
0.0 \\
0.0 \\
16679.051 \\
16673.297 \\
16677.660 \\
16676.932 \\
16676.179 \\
0 .\end{array}$ \\
\hline
\end{tabular}

and the complete analysis $\left[{ }^{4} \Pi(a) \rightarrow{ }^{4} \Sigma^{-}(a)\right]$. In the first of these stages, the molecular constants for each individual ${ }^{4} \Pi_{n}$ and ${ }^{4} \Sigma_{\Omega}^{-}$substate are calculated from combination relations. This is necessary to confirm the relationship between the various subbands, and also accentuates the differences between individual subband constants. Additionally, the recognition of individual subbands by other workers necessitates listing their separate constants. 


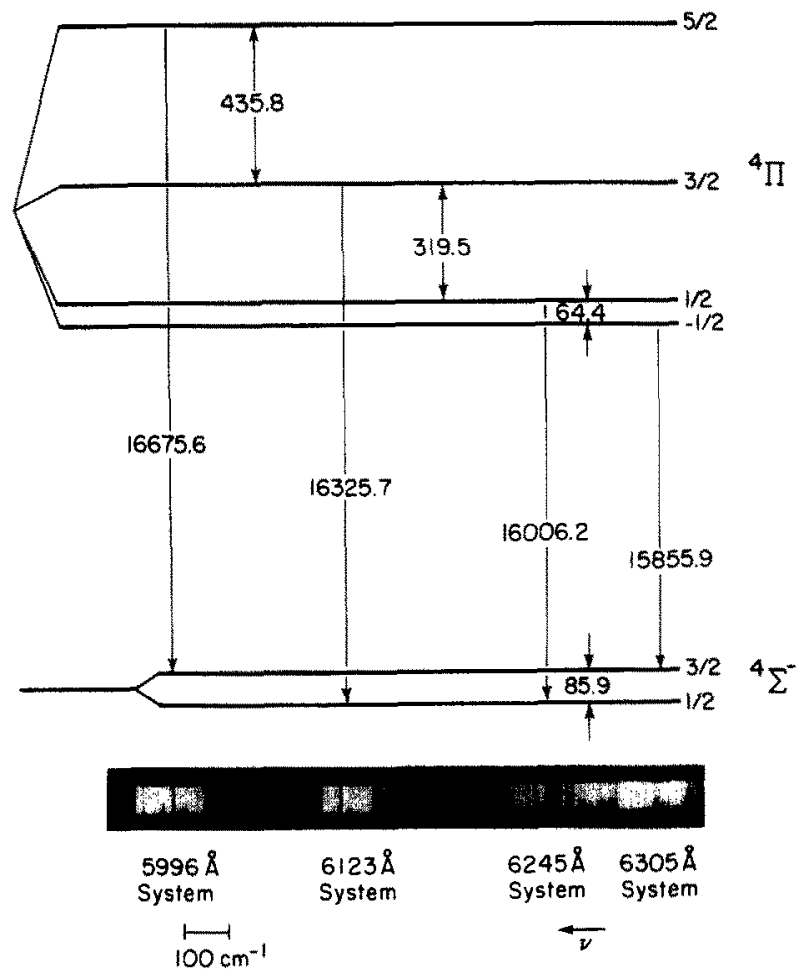

FiG. 1. Low-resolution spectrum and energy level diagram for the ${ }^{4} \Pi(a) \rightarrow{ }^{4}(a)$ transition in MoN.

In the second stage, the molecular constants for the ${ }^{4} \Sigma^{-}(a)$ state are calculated from combination relations and, using these values, greatly improved values for the ${ }^{4} \Pi_{\Omega}$ substate constants are then obtained from direct fits to the frequencies of the corresponding súbbands. In the final stage, the 1003 line frequencies comprising all four subband systems are fitted to the corresponding differences between the eigenvalues of the ${ }^{4} \Pi(a)$ and ${ }^{4} \Sigma{ }^{-}(a)$ Hamiltonian matrices in order to calculate constants for the ${ }^{4} \Pi(a)$ state.

\section{${ }^{4} \Pi$ State}

The Hamiltonian for each vibrational level of a ${ }^{4} \Pi$ state may be written as (18)

$$
H={ }^{e} T_{v}+H_{\mathrm{so}}+H_{\mathrm{rot}}+H_{\mathrm{ss}}+H_{\mathrm{sr}}+H_{\mathrm{A}},
$$

where

$$
\begin{aligned}
{ }^{e} T_{v} & =\text { the electronic and vibrational energy } \\
H_{\mathrm{so}} & =A_{v} L_{z} S_{z}+1 / 2 A_{D_{v}}\left(\tilde{R}^{2} L_{z} S_{z}+L_{z} S_{z} \tilde{R}^{2}\right) .
\end{aligned}
$$

The first term in $H_{\text {so }}$ is the spin-orbit interaction, and the second term is its centrifugal distortion correction. 

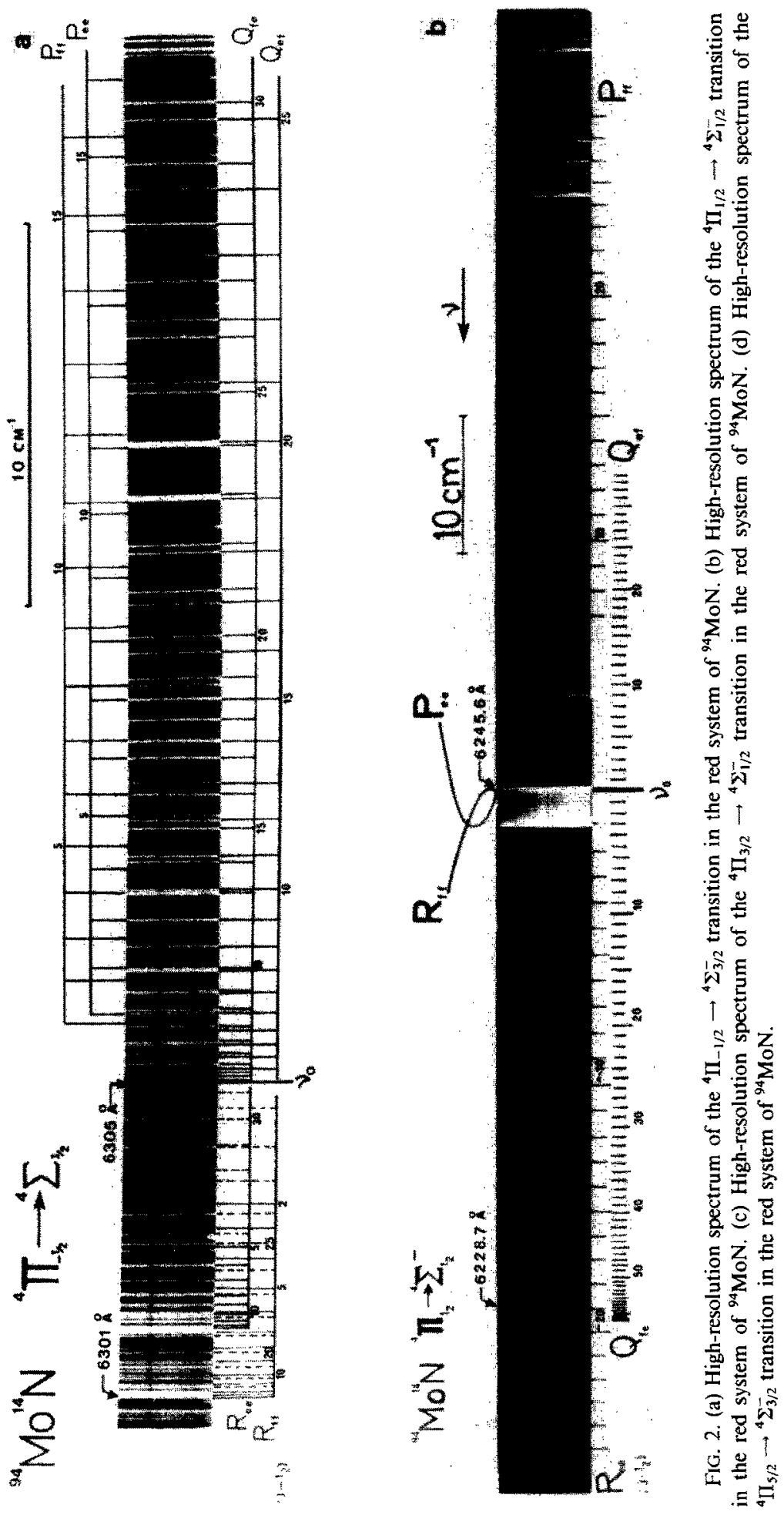
RED ELECTRONIC EMISSION SPECTRUM OF MON

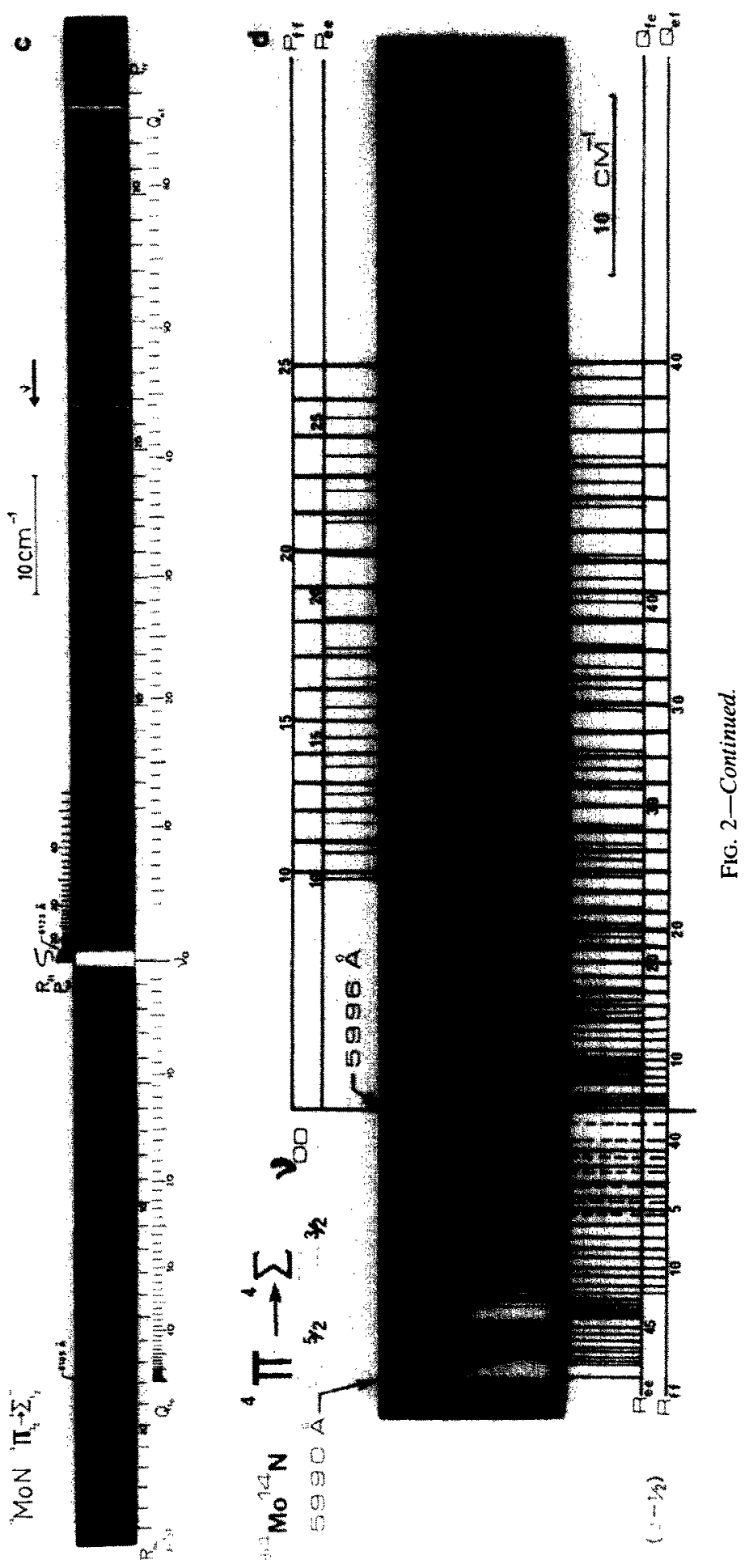




$$
H_{\text {rot }}=B_{v}(\tilde{J}-\tilde{L}-\tilde{S})^{2}-D_{v}(\tilde{J}-\check{L}-\check{S})^{4}
$$

$B_{v}$ is the rotational constant, and $D_{v}$ is its first centrifugal distortion correction.

$$
H_{\mathrm{ss}}=\epsilon_{v}\left(3 S_{z}^{2}-\tilde{S}^{2}\right)+1 / 2 \alpha_{v}\left(S_{+} S_{+}+S_{-} S_{-}\right) \delta_{0, \Delta \Omega}
$$

The first and second terms of $H_{\mathrm{ss}}$ are the parts of the spin-spin interaction which are diagonal and nondiagonal, respectively, in $\Lambda$.

$$
H_{\mathrm{sr}}=\gamma_{v}(\tilde{J}-\tilde{S}) \cdot \tilde{S}
$$

is the spin-rotation interaction diagonal in $\Lambda$.

$$
\begin{aligned}
H_{\Lambda}=1 / 2 q_{v}\left(J_{+}+J_{-}\right)^{2}+ & \left(1 / 2 q_{v}+1 / 2 p_{v}+o_{v}\right)\left(S_{+}+S_{-}\right)^{2} \\
& -\left(1 / 2 q_{v}+1 / 4 p_{v}\right)\left[\left(J_{+}+J_{-}\right)\left(S_{+}+S_{-}\right)+\left(S_{+}+S_{-}\right)\left(J_{+}+J_{-}\right)\right]
\end{aligned}
$$

is the lambda-doubling interaction. The lambda-doubling constants $p_{v}, q_{v}$, and $o_{v}$ are defined by Zare et al. (20). They show that $o_{v}$ cannot be obtained experimentally but, instead, is approximated by

$$
o_{v}=1 / 8\left(A_{v} / B_{v}\right) p_{v}
$$

The set of case (a) basis functions of definite parity $|\Omega \pm\rangle$ are, for a ${ }^{4} \Pi$ state,

$$
\begin{aligned}
|-1 / 2 \pm\rangle & =2^{-1 / 2}[|1,-3 / 2\rangle \pm|-1,3 / 2\rangle] \\
|1 / 2 \pm\rangle & =2^{-1 / 2}[|1,-1 / 2\rangle \pm|-1,1 / 2\rangle] \\
|3 / 2 \pm\rangle & =2^{-1 / 2}[|1,1 / 2\rangle \pm|-1,-1 / 2\rangle] \\
|5 / 2 \pm\rangle & =2^{-1 / 2}[|1,3 / 2\rangle \pm|-1,-3 / 2\rangle]
\end{aligned}
$$

The plus and minus signs refer to the $e$ and $f$ rotational levels, respectively, where the $e, f$ notation is according to Kopp and Hougen, (22) i.e., that rotational levels with parity $+(-1)^{J-1 / 2}$ are designated " $e$ " and those with parity $-(-1)^{J-1 / 2}$ are designated " $f$." With (2) as the basis set, the ${ }^{4} \Pi$ Hamiltonian reduces to two $4 \times 4$ symmetric matrices, one for the $e$ and one for the $f$ levels.

In the MoN molecule, perturbations from one or more unidentified electronic states cause the energy differences between the ${ }^{4} \Pi$ substates to differ markedly from each other, as can be seen from Fig. 1. The states causing these dramatic shifts in the substate energies have not been identified and, therefore, cannot be characterized. Accordingly, the diagonal matrix elements in (18) were modified in an empirical manner in order to take into account the effects of these perturbations. This was accomplished by replacing ${ }^{e} T_{v}$ in each diagonal element by $T_{\mathrm{n}}(\Omega=-1 / 2,1 / 2,3 / 2$, $5 / 2$ ), where $T_{\Omega}$ is a constant term which is different for each substate. The four $T_{\Omega}$ 's become adjustable parameters in the nonlinear least-squares fit of the eigenvalue differences to the 1003 line frequencies, as described below. The modified elements of the ${ }^{4} \Pi$ matrix are given in Table II.

\section{${ }^{4} \Sigma^{-}$State}

The Hamiltonian for each vibrational level of a ${ }^{4} \Sigma^{-}$state can be written as $(16,17)$ 
TABLE II

Modified Matrix Elements of the ${ }^{4} \Pi$ Hamiltonian in a Parity Case (a) Basis Set

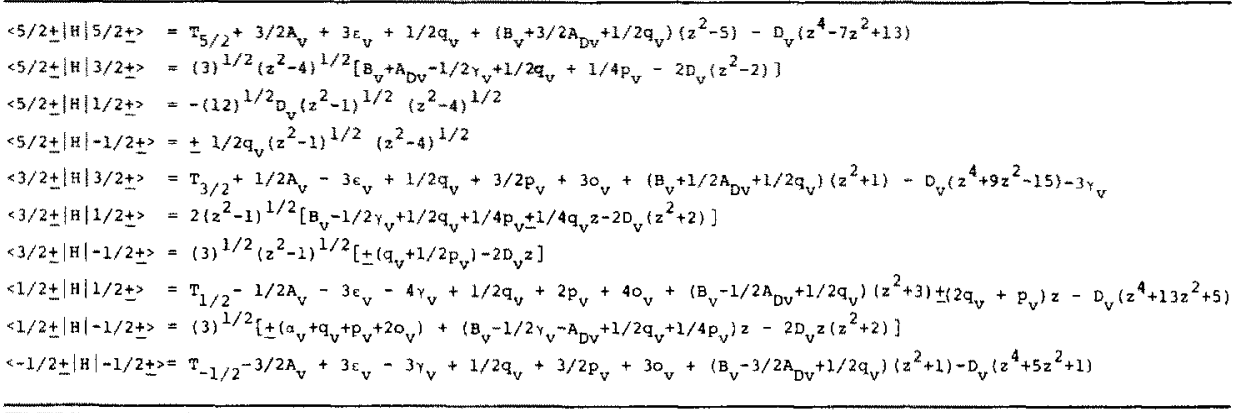

$$
H={ }^{e} T_{v}+H_{\text {rot }}+H_{\mathrm{ss}}+H_{\mathrm{sr}}+H_{\mathrm{so}}
$$

${ }^{e} T_{v}, H_{\text {rot }}$, and $H_{\mathrm{sr}}$ have the same meaning as for a ${ }^{4} \Pi$ state.

$$
H_{s s}=2 / 3 \lambda\left(3 S_{z}^{2}-\tilde{S}^{2}\right)
$$

is that part of the spin-spin interaction which is diagonal in $\Lambda$, and

$$
H_{\mathrm{so}}=\sum_{i} a_{i} \vec{l}_{i} \cdot \vec{s}_{i}
$$

is the second-order spin-orbit interaction and is not diagonal in $\Lambda$ or $\mathrm{S}$.

The parity case (a) basis functions $|\Omega \pm\rangle$ for a ${ }^{4} \Sigma^{-}$state, are

$$
\begin{aligned}
& |1 / 2 \pm\rangle=2^{-1 / 2}[|0,1 / 2\rangle \pm|0,-1 / 2\rangle] \\
& |3 / 2 \pm\rangle=2^{-1 / 2}[|0,3 / 2\rangle \pm|0,-3 / 2\rangle]
\end{aligned}
$$

Using this basis set the $4 \times 4^{4} \Sigma^{-}$Hamiltonian matrix reduces to two $2 \times 2$ matrices, one for the $e$ and one for the $f$ rotational levels. The matrix elements are given in Table III. The $2 \times 2$ matrices are easily diagonalized analytically to obtain their eigenvalues, which are, respectively, the $e$ and $f$ rotational levels of the ${ }^{4} \Sigma_{1 / 2}^{-}$ and ${ }^{4} \Sigma_{3 / 2}^{-}$substates.

The spin-spin and spin-rotation interactions have the same quantum number dependence as the second-order spin-orbit interaction $(16,17)$. In addition, the spin-spin and spin-rotation parameters are considerably smaller than the contributions to the eigenvalues from the off-diagonal elements of the spin-orbit interaction. Thus, the parameter $\lambda$ can be considered as an "effective" spin-splitting parameter; most of the contributions to it are from the off-diagonal spin-orbit effects. $\lambda$ is defined so that ${ }^{e} T_{0}\left({ }^{4} \Sigma_{3 / 2}^{-}\right)-{ }^{e} T_{0}\left({ }^{4} \Sigma_{1 / 2}^{-}\right)=4 \lambda$.

Preliminary Analysis (Subband by Subband): ${ }^{4} \Pi_{\Omega} \rightarrow{ }^{4} \Sigma_{\Omega}^{-}$

On the assumption of the transition as ${ }^{4} \Pi(a) \rightarrow{ }^{4} \Sigma^{-}(a)$ and utilizing the usual selection rules, the energy level diagram is as given in Fig. 3. 
Matrix Elements of the ${ }^{4} \Sigma$ Hamiltonian in a Parity Case $(a)$ Basis Set

$$
\begin{aligned}
& <1 / 2 \pm|H| I / 2 \pm>=T_{0}-2 \lambda+\left(B-4 \lambda_{J}\right)(Z+4) \\
& \mp 2\left(B-4 x_{J}-1 / 2 \gamma_{2}\right)(J+1 / 2) \\
& -D\left((z+4)^{2}+3 z+4(J+1 / 2)^{2}\right. \\
& \left.\mp^{4}(z+4)(J+1 / 2)\right) \\
& \langle 3 / 2 \pm|H| 3 / 2 \pm\rangle=T_{0}+2 \lambda+\left(B+4 \lambda_{J}\right) Z-D\left(Z^{2}+3 Z\right) \\
& \langle 1 / 2 \pm|H| 3 / 2 \pm\rangle=-(37)^{1 / 2}\left[B-1 / 2 \gamma_{1}-D\left\{2(Z+4) z^{2} 2(J+1 / 2)\right\}\right] \\
& Z=(J-1 / 2)(J+3 / 2)
\end{aligned}
$$

As is shown by Kopp and Hougen (25), the rotational levels for a ${ }^{2 S+1} \Sigma_{1 / 2}(a)$ state are approximately expressible as

$$
F_{1 / 2, e}(\mathrm{~J})=B_{1 / 2} J(J+1) \mp 1 / 2 p_{1 / 2}(J+1 / 2)
$$

where the $\Omega$-doubling constant $p=\mp(-1)^{S+1 / 2}(2 S+1) B_{1 / 2}$ for $\Lambda=0^{ \pm}$, and $p=0$ for $\Lambda \neq 0$. (For a ${ }^{4} \Sigma_{1 / 2}$ state, $p=+4 B_{1 / 2}$.) Similarly, the approximate formula for the ${ }^{4} \Pi(a)$ state may be written (27) (for $A>0$ )

$$
\underset{\Omega}{F_{\Omega, e}}(J)=B_{\Omega} J(I+1) \pm 1 / 2 p_{\Omega}(I+1 / 2) \mp \cdots,
$$

where $p_{\mathfrak{n}}$ is the lambda-doubling constant. When the line frequencies of the six branches $Q_{e f}, Q_{f e}, \ldots, R_{e e}$ are calculated using Eqs. (5) and (6), assuming that

$$
\begin{aligned}
& \text { (i) } B_{\Omega}^{\prime} \sim B_{\Omega}^{\prime \prime} \sim B \\
& \text { (ii) } p_{\Omega}^{\prime \prime}=\left(p_{1 / 2}^{\prime \prime}\right) \sim 4 B_{\Omega}^{\prime \prime} \sim 4 B \\
& \text { (iii) } p_{\Omega}^{\prime \prime} \gg p_{\Omega}^{\prime}
\end{aligned}
$$

spacings of $\mathrm{OB}, \pm 2 B$, and $\pm 4 B$ are obtained for these branches, which terminate upon the ${ }^{4} \Sigma_{1 / 2}^{-}$substate. It may easily be verified that branches terminating upon the ${ }^{4} \Sigma_{3 / 2}^{-}$substate have only $0 B$ and $\pm 2 B$ spacings with the same assumptions.

(a) $T h e^{4} \Sigma^{-}$state. One can generalize the Kopp and Hougen equation (5) as follows:

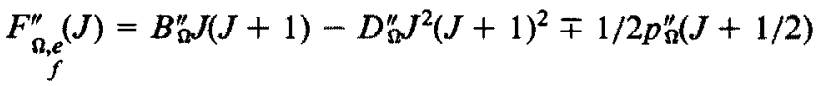

$$
\begin{aligned}
& \pm 1 / 2 p_{J, \Omega}^{\prime \prime}(J+1 / 2)^{3} \mp 1 / 2 p_{J,, \Omega}^{\prime \prime}(J+1 / 2)^{5} \pm \cdots,
\end{aligned}
$$




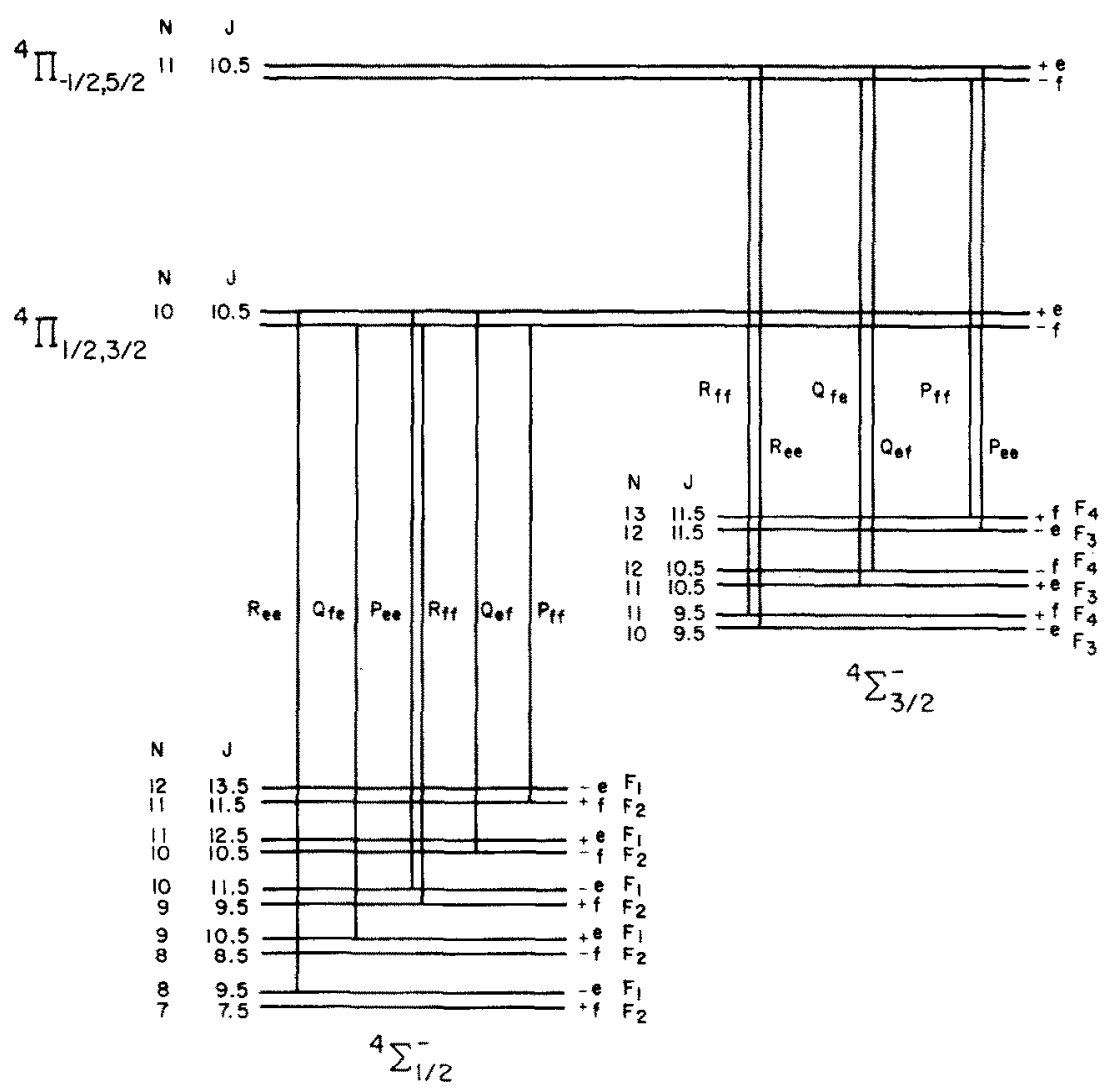

Fig. 3. Rotational energy level diagram for a $\mathrm{A}(a) \rightarrow{ }^{4} \Sigma^{-}(a)$ transition. Note that the upper set of levels refers to the two components ${ }^{4} \Pi_{-1 / 2}$ and ${ }^{4} \Pi_{5 / 2}$ while the lower is for ${ }^{4} \Pi_{1 / 2}$ and ${ }^{4} \Pi_{3 / 2}$.

where $p_{J, \Omega}^{\prime \prime}$ and $p_{J J, \Omega}^{\prime \prime}$ are higher-order $\Omega$-doubling constants. Reference to Fig. 3 enables the combination relations $(A 2)$ given in the Appendix to be fitted to polynomials of the second degree in $(J+1)^{2}$, yielding $B_{\Omega}^{\prime \prime}$ and $D_{\Omega}^{\prime \prime}$ as given in Table $\mathrm{V}$.

(b) The ${ }^{4} \Pi$ state. Generalizing Eq. (6) yields (for $A>0$ )

$$
\begin{aligned}
F_{\mathrm{a}, e}^{\prime}(J)=B_{0}^{\prime} J(J+1)-D_{\Omega}^{\prime} J^{2}(J+1)^{2} & \pm 1 / 2 p_{\Omega}^{\prime}(J+1 / 2) \\
& \mp 1 / 2 p_{J, \Omega}^{\prime}(J+1 / 2)^{3} \pm 1 / 2 p_{J, \Omega \Omega}^{\prime}(J+1 / 2)^{5} \mp \cdots .
\end{aligned}
$$

As in the case of the ${ }^{4} \Sigma^{-}$state, fits to suitable combination relations (A4) enable the analogous ${ }^{4} \Pi_{\Omega}$ constants to be calculated. These are given in Table IV.

Examination of Table IV shows that the $B_{\Omega}^{\prime}$ and $D_{\Omega}^{\prime}$ values have no obvious pattern, the $D^{\prime}$ value for $\Omega=-1 / 2$ being, apparently, negative. Nor can the constants be fitted to a relationship of the form known for ${ }^{2} \Pi$ and ${ }^{3} \Pi$ states, i.e.,

$$
B_{\mathrm{\Omega}}=B_{v}\left(1+\frac{2 \Sigma B_{v}}{A \Lambda}+\cdots\right)
$$


TABLE IV

Molecular Constants for the ${ }^{4} \Pi_{\Omega}$ Substates Obtained from Least-Squares Fits to Combination Differences of Each Subband $\left(\mathrm{cm}^{-1}\right)$

\begin{tabular}{l|c|c|c|c|c}
\hline Substate & $D$ & $D$ & $D$ & $P_{J}$ & $F_{J J}$ \\
\hline $4_{\pi}$ & 0.49400 & $-2.29 \times 10^{-7}$ & -0.17356 & $-5.20 \times 10^{-5}$ & $-9.15 \times 10^{-9}$ \\
\hline $4_{\pi_{1 / 2}}$ & 0.51041 & $1.13 \times 10^{-6}$ & 0.22549 & $4.86 \times 10^{-5}$ & $6.76 \times 10^{-9}$ \\
\hline $4_{\pi_{3 / 2}}$ & 0.50532 & $5.46 \times 10^{-7}$ & $1.9 \times 10^{-5}$ & $-7.8 \times 10^{-7}$ & $-5.2 \times 10^{-11}$ \\
\hline $4_{\pi_{5 / 2}}$ & 0.50593 & $4.14 \times 10^{-7}$ & $-2.3 \times 10^{-4}$ & $-7.7 \times 10^{-7}$ & $-3.6 \times 10^{-10}$ \\
\hline
\end{tabular}

where the symbols have their usual significance. Thus, Table IV shows that a value of $B_{v}$ cannot be obtained from the $B_{\Omega}$ 's and their appropriate $\Sigma$ values, unless the higher terms in the expression, which are usually small, are unexpectedly large.

The constants in Table IV and V, along with the subband origins, $\nu_{0}$, were then used to calculate the frequencies of the six branches of each of the ${ }^{4} \Pi_{5 / 2} \rightarrow{ }^{4} \Sigma_{3 / 2}^{-}$, ${ }^{4} \Pi_{3 / 2} \rightarrow{ }^{4} \Sigma_{1 / 2}^{-},{ }^{4} \Pi_{1 / 2} \rightarrow{ }^{4} \Sigma_{1 / 2}^{-}$, and ${ }^{4} \Pi_{-1 / 2} \rightarrow{ }^{4} \Sigma_{3 / 2}^{-}$transitions. The origins, $\nu_{0}$, were obtained from fits of $Q_{e f}(J)+Q_{f e}(J)$ to polynomials of third degree in $J$. In all four subbands the calculated frequences agree with their measured counterparts to within $\sim 0.2 \mathrm{~cm}^{-1}$ for small values of $J$. However, as $J$ increases to 60.5 the differences become as large as $1 \mathrm{~cm}^{-1}$ for the $6305-\AA$ subband, $8 \mathrm{~cm}^{-1}$ for the 6245 - and 5996 $\AA$ subbands, and $12 \mathrm{~cm}^{-1}$ for the $6123-\AA$ subband!

TABLE $V$

${ }^{4} \mathrm{Z}^{-}$Molecular Constants Obtained from Least-Squares Fits to Combination Differences $\left(\mathrm{cm}^{-1}\right)$

\begin{tabular}{|c|c|c|c|c|c|c|}
\hline & \multicolumn{2}{|c|}{${ }^{4} \varepsilon_{1 / 2}^{-}$} & \multicolumn{2}{|c|}{${ }^{4} \Sigma_{3 / 2}^{-}$} & \multicolumn{2}{|c|}{${ }^{4} \Sigma^{-}$} \\
\hline & ${ }^{4} n_{1 / 2}+{ }^{4} \bar{\Sigma}_{1 / 2}^{-}$ & ${ }^{4} \pi_{3 / 2}+{ }^{4} \varepsilon_{1 / 2}^{-}$ & ${ }^{4} \pi_{-1 / 2}+{ }^{4} \Sigma_{3 / 2}^{-}$ & ${ }^{4} \pi_{5 / 2} \cdot{ }^{4} \varepsilon_{3 / 2}^{-}$ & & a) ${ }^{4} \Sigma^{-}(a)$ \\
\hline B & 0.50753 & 0.50722 & 0.52659 & 0,52643 & 3 & $0.51679(45)$ \\
\hline $\mathbf{v}$ & $4.24 \times 10^{-7}$ & $1.01 \times 10^{-7}$ & $5,42 \times 10^{-7}$ & $7.74 \times 10^{-7}$ & D & $4.92(11) \times 10^{-7}$ \\
\hline p & 2.0407 & 2.0360 & $1.52 \times 10^{-3}$ & $4.94 \times 10^{-3}$ & $\lambda$ & $23.466(8)$ \\
\hline$P_{J}$ & $2.15 \times 10^{-4}$ & $2.01 \times 10^{-4}$ & $-2.18 \times 10^{-4}$ & $-2.04 \times 10^{-4}$ & $\gamma$ & $0.0109(20)$ \\
\hline $\mathbf{p}_{\mathrm{JJ}}$ & $3.1 \times 10^{-8}$ & $2.4 \times 10^{-8}$ & $-3.3 \times 10^{-8}$ & $-2.6 \times 10^{-8}$ & $\lambda_{J}$ & $-7.1(15) \times 10^{-6}$ \\
\hline$\dot{0}\left(p_{*} p_{J}\right)$ & $6.7 \times 10^{-5}$ & $9.4 \times 10^{-5}$ & $1.7 \times 10^{-4}$ & $1.4 \times 10^{-4}$ & $\dot{\sigma}$ & 0.017 \\
\hline$\hat{\sigma}(B$ and $D)$ & $5.1 \times 10^{-4}$ & $2.5 \times 10^{-3}$ & $8.5 \times 10^{-4}$ & $2.7 \times 10^{-3}$ & & \\
\hline
\end{tabular}


The observed lambda doubling, $\Delta \nu_{\Omega}^{\mathrm{EXP}}(\mathrm{J})=F_{\Omega, e}^{\prime}(J)-F_{\Omega, f}^{\prime}(J)$, of the ${ }^{4} \Pi_{-1 / 2}$, ${ }^{4} \Pi_{1 / 2}$, and ${ }^{4} \Pi_{3 / 2}$ substates is shown in Fig. 4. There is no observable splitting of the ${ }^{4} \Pi_{5 / 2}$ substate. According to the Budo and Kovacs formulae (28), the splitting of the $-1 / 2$ substate is negative and is proportional to $J$. The splitting of the $1 / 2$ substate is positive, is also proportional to $J$, and is comparable in magnitude to that of the $-1 / 2$ substate. The splittings of the $3 / 2$ and $5 / 2$ substates are much smaller and are proportional to $J^{3}$ and $J^{5}$, respectively. An attempt was made to fit the observed lambda doubling of the four substates to the Budo and Kovacs expressions. These expressions involve the constants $C_{i}(i=0,1,2)$ together with $Y(=A / B)$ and were evaluated from fits to combination relations (A4), i.e., since $\Delta \nu_{\Omega}(J)=F_{\Omega, e}^{\prime}(J)-F_{\Omega, f}^{\prime}(J)$.

$$
\begin{aligned}
& \frac{\Delta_{1}^{a} F_{-1 / 2}^{\prime}(J)-\Delta_{1}^{b} F_{-1 / 2}^{\prime}(J)}{4(J+1)}=-\frac{\sqrt{3} C_{0}}{Y+2} \\
& \frac{\Delta_{1}^{a} F_{1 / 2}^{\prime}(J)-\Delta_{1}^{b} F_{1 / 2}^{\prime}(J)}{4(J+1)}= \frac{\sqrt{3} C_{0}+C_{1}(Y+2)}{Y+2} \\
& \frac{\Delta_{1}^{a} F_{3 / 2}^{\prime}(J)-\Delta_{1}^{b} F_{3 / 2}^{\prime}(J)}{4(J+1)}= \frac{2 \sqrt{3} C_{0}+C_{1}(7 Y-6)+2 C_{2} Y(Y-2)}{Y(Y-2)^{2}} \times\left(J+\frac{1}{2}\right)\left(J+\frac{3}{2}\right) \\
& \frac{\Delta_{1}^{a} F_{5 / 2}^{\prime}(J)-\Delta_{1}^{b} F_{5 / 2}^{\prime}(J)}{4(J+1)}= \frac{\sqrt{3} C_{0}+6 C_{1}(Y-3)+4 C_{2}(Y-3)(Y-4)}{(Y-2)(Y-4)^{2}(Y-6)^{2}} \\
& \quad \times\left(J-\frac{1}{2}\right)\left(J+\frac{1}{2}\right)\left(J+\frac{3}{2}\right)\left(J+\frac{5}{2}\right) \quad(10)
\end{aligned}
$$

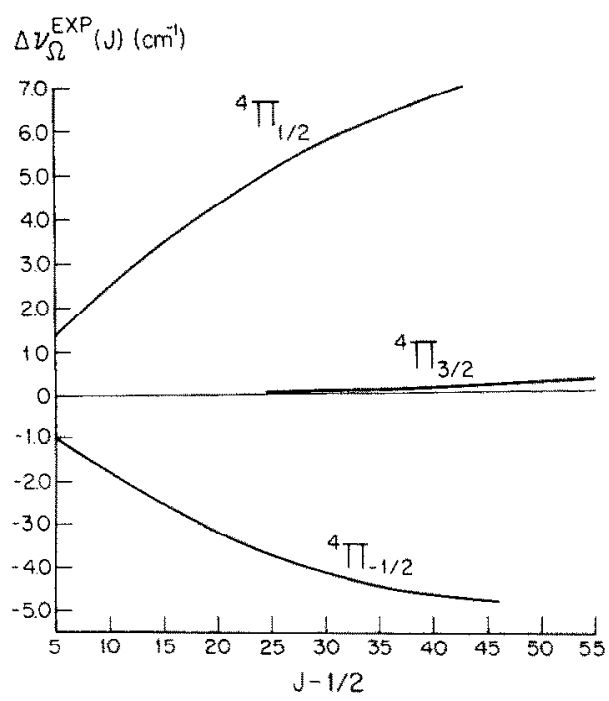

Fig. 4. The observed lambda-doubling of the ${ }^{4} \Pi(a)$ state of MoN. Note that no doubling was observed for ${ }^{4} \Pi_{5 / 2}$ and that this, therefore, is not included in the figure. 
All four substates were included in the fit, which was carried out using the program MARQ (29), which utilizes Marquardt's method of nonlinear least squares (30). The results, which are presented in Table VI, are poor. The differences between calculated and observed combination differences exceed $100 \%$ for large values of $J$. The value of $Y$, which yields $A_{v} \sim 220 \mathrm{~cm}^{-1}$, is at least of the correct order of magnitude. A repeat of the fit in which the ${ }^{4} \Pi_{5 / 2}$ substate was omitted produced even worse results. This discrepancy is not surprising however, since, as shown in Fig. 4, the observed splittings are not in agreement with the Budo-Kovacs predictions.

\section{The Intermediate Analysis: ${ }^{4} \Pi_{\Omega} \rightarrow{ }^{4} \Sigma^{-}(a)$}

The molecular constants $B, D, \lambda, \gamma$, and $\lambda_{J}$ for the ${ }^{4} \Sigma^{-}(a)$ state were obtained by using program MARQ to perform a nonlinear least-squares fit of sums and differences of the eigenvalues of the ${ }^{4} \Sigma^{-}$matrix (Table III) to observed combination differences. $\left(\gamma_{1}\right.$ is placed equal to $\gamma_{2}$, and both are denoted by $\gamma$.) According to Eqs. (A1) and (A2), for each ${ }^{4} \Sigma_{\Omega}$ substate:

$$
\frac{\Delta_{1}^{a} F_{\Omega}^{\prime \prime}(J)+\Delta_{1}^{b} F_{\Omega}^{\prime \prime}(J)}{2}=F_{\Omega, f}^{\prime \prime}(J)+F_{\Omega, f}^{\prime \prime}(J+1)-F_{\Omega, e}^{\prime \prime}(J)-F_{\Omega, e}^{\prime \prime}(J+1)
$$

A total of 153 combination differences, encompassing all four ${ }^{4} \Pi_{\Omega} \rightarrow{ }^{4} \Sigma_{\Omega}^{-}$subbands, were included in the fit. The values of the ${ }^{4} \Sigma^{-}(a)$ constants which resulted are given in Table $\mathrm{V}$.

Figure 5 is the theoretical spin-splitting diagram of the ${ }^{4} \Sigma^{-} v=0$ state of MoN. The calculated term values, $F_{\mathrm{i}}^{\prime \prime}(N)$, minus the purely rotational energy, $B^{\prime \prime} N(N+1)$ $-D^{\prime \prime} N^{2}(N+1)^{2}$, are plotted against $N$. The term values are the eigenvalues of the ${ }^{4} \boldsymbol{\Sigma}^{-}$matrix (Table III), using the molecular constants in Table V. The usual relationships hold between the $F_{\mathrm{i}}^{\prime \prime}, N$, and $J$, i.e., $F_{1}^{\prime \prime}: J=N+3 / 2 \cdots F_{4}^{\prime \prime}$ : $J=N-3 / 2$.

Figure 5 shows the transition of the ${ }^{4} \Sigma$ state from Hund's case (a) to case (b) as $N$ increases beyond $\sim 75$. A ${ }^{4} \Sigma$ state is better represented by a case (a) basis than by a case (b) basis when $|4 \lambda|>\sqrt{3} B J(16)$. A case (a) state comprises two substates, ${ }^{4} \Sigma_{1 / 2}$ and ${ }^{4} \Sigma_{3 / 2}$, which are separated in energy at low values of $J$ by $4 \lambda$.

\section{TABLE VI}

Lambda-Doubling Constants for the ${ }^{4} \Pi$ State Obtained by Fitting Combination Differences to the Formulae of Budo and Kovacs $\left(\mathrm{cm}^{-1}\right)$

$$
\begin{aligned}
& Y=425 * \\
& C_{0}=31 \\
& C_{1}=0.034 \\
& C_{2}=-4.3 \times 10^{-4} \\
& * Y=A / B
\end{aligned}
$$


As was shown above, the line frequencies calculated using the molecular constants obtained from combination differences disagree markedly with the measured frequencies at large values of $J$. Therefore, improved sets of constants for the ${ }^{4} \Pi_{\Omega}$ substates were obtained by using MARQ to fit differences between calculated ${ }^{4} \Pi_{\Omega}$ and ${ }^{4} \Sigma^{-}(a)$ rotational levels directly to the corresponding frequencies. The ${ }^{4} \Pi_{\Omega}$ energy level expressions used are the same as before, i.e., Eq. (8), but the ${ }^{4} \Sigma^{-}(a)$ formulae are now the matrix eigenvalues (Table III). In addition, the ${ }^{4} \Sigma^{-}(a)$ constants are not allowed to vary during the fit, but, instead, are fixed to the values in Table $\mathrm{V}$. The reason for this is that the ${ }^{4} \Sigma^{-}(a)$ eigenvalues are derived using a very accurate Hamiltonian, Eq. (3), whereas the ${ }^{4} \Pi_{\Omega}$ expressions, Eq. (8), are very simplistic.

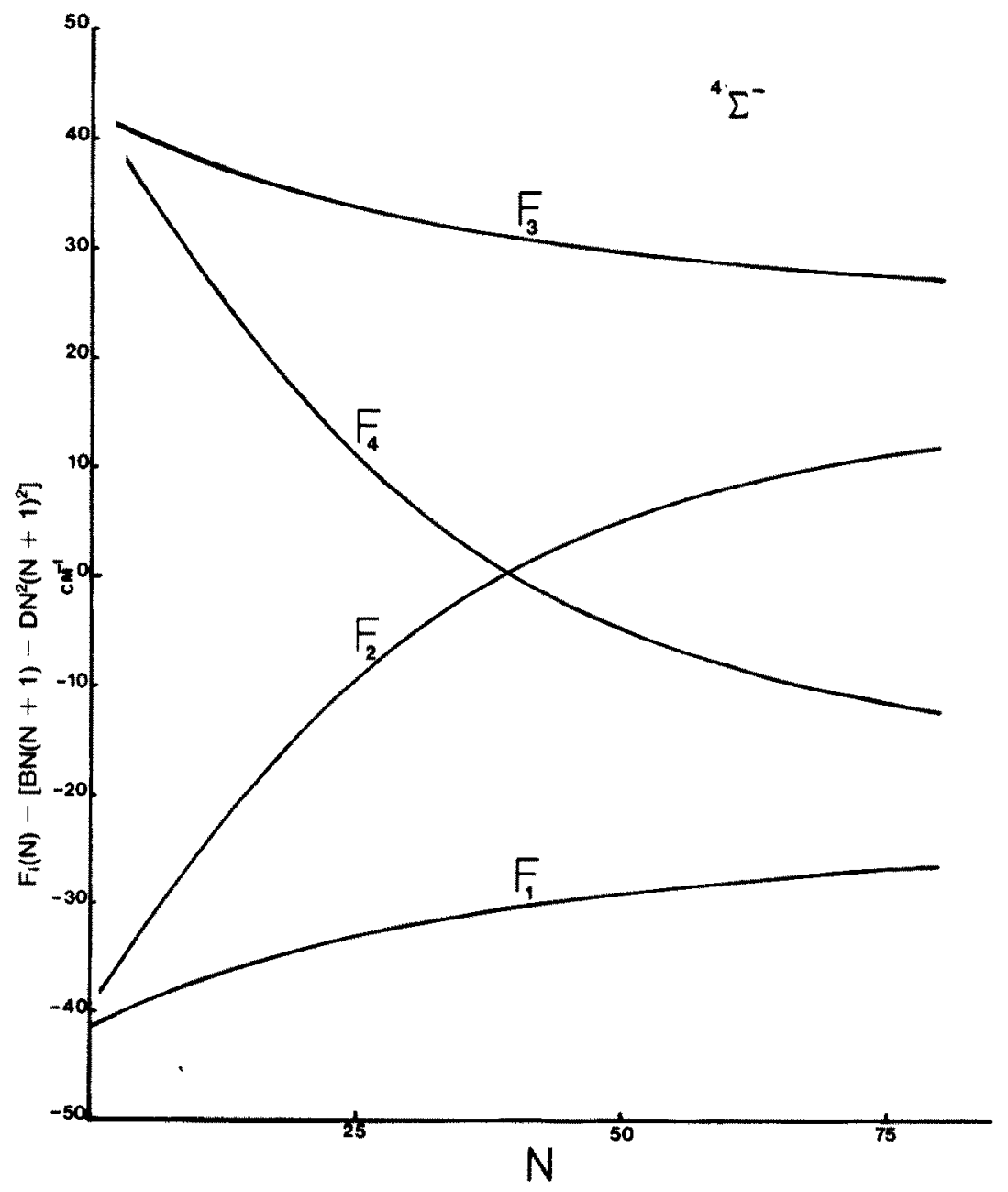

FIG. 5. Theoretical spin splitting diagram for the ${ }^{4} \Sigma^{-}(a) v=0$ state. Note that the figure has been drawn to correspond to the calculated levels for the ${ }^{4} \boldsymbol{\Sigma}^{-}(a)$ state of MoN. 
MARQ is used to fit the differences between the rotational levels of the ${ }^{4} \Pi_{\Omega}$ and ${ }^{4} \Sigma_{\Omega}^{-}$substates to the measured frequencies of each subband to obtain $B_{\Omega}^{\prime}, D_{\Omega}^{\prime}, p_{\Omega}^{\prime}, p_{J, \Omega}^{\prime}$, and $p_{J J, \Omega}^{\prime}$. The results are summarized in Table VII. The fits for the ${ }^{4} \Pi_{3 / 2} \rightarrow{ }^{4} \Sigma_{1 / 2}^{-}$and ${ }^{4} \Pi_{5 / 2} \rightarrow{ }^{4} \Sigma_{3 / 2}^{-}$subbands are extremely good, as can be seen from the values of the standard deviations $\left(\sim 0.01 \mathrm{~cm}^{-1}\right)$. In fact, the maximum deviation between measured and calculated frequencies is $\approx 0.03 \mathrm{~cm}^{-1}$. The fits for the ${ }^{4} \Pi_{1 / 2} \rightarrow{ }^{4} \Sigma_{1 / 2}^{-}$and ${ }^{4} \Pi_{-1 / 2} \rightarrow{ }^{4} \Sigma_{3 / 2}^{-}$subbands are not quite as good; the standard deviations are $\approx 0.04 \mathrm{~cm}^{-1}$. In the latter two subbands, there are branches whose calculated frequencies differ from the measured values by as much as $0.20 \mathrm{~cm}^{-1}$ for values of $J$ between 55.5 and 60.5 .

\section{The Concerted Analysis: ${ }^{4} \Pi(a) \rightarrow{ }^{4} \Sigma^{-}(a)$}

The molecular constants for the ${ }^{4} \Pi$ state taken as a whole are obtained from the "direct" nonlinear least-squares fitting procedure of Zare et al. (20). In this procedure the Hamiltonian matrices of the upper and lower electronic states are numerically diagonalized, and the differences between the eigenvalues are fitted directly to the corresponding measured line frequencies. This approach allows all the observed lines (except those deliberately rejected) to be included in the fit, regardless of how fragmentary a given branch may be. A program named DIRECT was written to calculate the ${ }^{4} \Pi$ constants by calling MARQ to perform the fit.

In the case of $a^{4} \Sigma^{-}(a)$ state it is not necessary to numerically diagonalize the Hamiltonian matrix, since, in the case (a) parity basis set (4), it reduces to two $2 \times 2$ matrices. DIRECT first calculates the ${ }^{4} \Sigma^{-}$eigenvalues, $F_{\Omega, e}^{\prime \prime}(J)$ and $F_{\Omega, f}^{\prime \prime}(J)$, using the ${ }^{4} \Sigma^{-}(a)$ molecular constants, obtained from the combination relations, in Table V. As in the previous calculation, these eigenvalues remain unchanged during the fit, i.e., the ${ }^{4} \Sigma^{-}(a)$ constants are not varied by MARQ. In view of the strong perturbations acting upon the ${ }^{4} \Pi$ state, it was thought worthwhile to omit the spinorbit splitting constant, $A$, altogether. This was accomplished by fixing the value of $A$ to zero during the fit. The ${ }^{4} \Pi$ constants which result are given in Table VIII.

\section{TABLE VII}

Molecular Constants for the ${ }^{4} \Pi_{\Omega}$ Substates Obtained from Least-Squares Fits Directly to the Frequencies of Each Subband $\left(\mathrm{cm}^{-1}\right)$ The lower state used is the ${ }^{4-}$ matrix (Table III) with fixed values of the ${ }^{4} \Sigma^{-}$ constants (Table V)]*

\begin{tabular}{|c|c|c|c|c|c|c|}
\hline Substates & D & $D$ & $p$ & $\mathrm{P}_{3}$ & $P_{\mathrm{JJ}}$ & $\hat{a}$ \\
\hline${ }^{4} \pi-1 / 2$ & $0.49360(1)$ & $-1.49(3) \times 10^{-7}$ & $-0.16974(39)$ & $-4.21(6) \times 10^{-5}$ & $-4.61(24) \times 10^{-9}$ & 0.041 \\
\hline${ }^{4} \pi_{1 / 2}$ & $0.50995(1)$ & $9.47(1) \times 10^{-7}$ & $0.22438(32)$ & $4.57(3) \times 10^{-5}$ & $5.55(8) \times 10^{-9}$ & 0.041 \\
\hline $4_{3 / 2}$ & $0.50506(1)$ & $5.13(1) \times 10^{-7}$ & $4.4(31) \times 10^{-4}$ & $-1.3131 \times 10^{-6}$ & $-1,9(80) \times 10^{-11}$ & 0.012 \\
\hline${ }^{4} n_{5 / 2}$ & $0.50591(1)$ & $4.79(1) \times 10^{-7}$ & & & & 0.009 \\
\hline
\end{tabular}

The uncertainties listed in this table do not include any contribution from the constants of the quartet sigma (ground) state. Inclusion of them increases the magnitude of the uncertainties by about an additional factor of two. 
TABLE VIII

${ }^{4} \Pi$ Constants Obtained from a Least-Squares Fit Directly to the Frequencies of the 0,0 Band (All constants in $\mathrm{cm}^{-1}$ )

\begin{tabular}{lc}
\hline$T_{-1 / 2}$ & $15,898.84$ \\
$T_{1 / 2}$ & $15,963.26$ \\
$T_{3 / 2}$ & $16,282.73$ \\
$T_{5 / 2}$ & $16,718.48$ \\
$A$ & 0.0 (fixed \\
$B$ & 0.50380 \\
$E$ & -0.252655 \\
$a$ & 2.0096 \\
$p$ & 0.025970 \\
$q$ & $-1.9445 \times 10^{-4}$ \\
$\gamma$ & 0.074130 \\
$A_{D}$ & $4.0295 \times 10^{-4}$ \\
$D$ & $4.8072 \times 10^{-7}$ \\
$\hat{\theta}$ & 0.11 \\
\hline
\end{tabular}

The standard deviation of the fit is $\sim 0.10 \mathrm{~cm}^{-1}$, and the differences between the measured and calculated frequencies vary from $\sim 0.01$ to $0.6 \mathrm{~cm}^{-1}$. As is noted in the next section, nearly all of the discrepancies arise from the failure of the perturbation Hamiltonian $H_{A}$ to describe the lambda doubling of the ${ }^{4} \Pi_{3 / 2}$ substate.

\section{DISCUSSION}

The remaining problems in the analysis of the MoN spectrum are: (i) Deciding the functional forms of the corrections to the diagonal elements of the ${ }^{4} \Pi$ matrices. The constants in Table VIII are calculated using only an empirical parameter, $T_{n}$, which is adjusted during the fitting in order to absorb the effects of the perturbations. (ii) Deriving corrections to the Hamiltonian for the lambda-doubling interaction, $H_{A}$, which, as it stands, fails to predict the lambda doubling observed in the ${ }^{4} \Pi_{3 / 2}$ substate.

In order to shed light on these problems the eigenvalues of the ${ }^{4} \Pi$ matrices, which are calculated by DIRECT, were compared with experimental values of the ${ }^{4} \Pi$ rotational energy levels. It is not possible in general to determine absolute experimental values of the rotational levels; one can only measure their differences, i.e., the frequencies. However, for the ${ }^{4} \Pi(a) \rightarrow{ }^{4} \Sigma^{-}(a)$ transition in MoN, this can be resolved by calculating "experimental" rotational levels for the ${ }^{4} \Pi$ state, which do not depend upon any theoretical model of that state and which include the 
electronic and vibrational energy, ${ }^{e} T_{0}$, of the state. These are denoted $T_{\Omega, e}^{\prime}(J)$ and $T_{\Omega, f}^{\prime}(J)$ and are given by Eqs. (12) and (13);

$$
\begin{aligned}
T_{\Omega, e}^{\prime}(J)=1 / 3\left[F_{\Omega, e}^{\prime \prime}(J-1)+F_{\Omega, f}^{\prime \prime}(J)+\right. & F_{\Omega, e}^{\prime \prime}(J+1) \\
& \left.+P_{e e, \Omega}(J+1)+Q_{e f, \Omega}(J)+R_{e e, \Omega}(J-1)\right] \\
T_{\Omega, f}^{\prime}(J)=1 / 3\left[F_{\Omega, f}^{\prime \prime}(J-1)+F_{\Omega, e}^{\prime \prime}(J)+\right. & F_{\Omega, f}^{\prime \prime}(J+1) \\
& \left.+P_{f, \Omega}(J+1)+Q_{f e, \Omega}(J)+R_{f f, \Omega}(J-1)\right] .
\end{aligned}
$$

The matrix isolation studies of Bates and Gruen (13) and Knight and Steadman (14) indicate that the ${ }^{4} \Sigma^{-}$state is the ground state of $\mathrm{MoN}$. The calculated rotational

\begin{tabular}{|c|c|c|c|c|c|c|}
\hline \multirow[b]{2}{*}{$\mathbf{J}$} & \multicolumn{3}{|c|}{ E LEVELS } & \multicolumn{3}{|c|}{ F LEVELS } \\
\hline & EXPTL. & CALCD. & DIFF. & EXPTL. & CALCD. & DIFF. \\
\hline 2.5 & 15902.650 & 15902.696 & -0.046 & 15903.172 & 15903.214 & -0.042 \\
\hline 3.5 & 906.022 & 906.063 & -0.041 & 906.717 & 906.752 & -0.035 \\
\hline 4.5 & 910.388 & 910.417 & -0.029 & 911.231 & 911.276 & -0.045 \\
\hline 5.5 & 915.709 & 915.759 & -0.050 & 916.754 & 916.785 & -0.031 \\
\hline 6.5 & 922.030 & 922.088 & -0.058 & 923.239 & 923.280 & -0.041 \\
\hline 7.5 & 929.348 & 929.405 & -0.057 & 930.727 & 930.761 & -0.034 \\
\hline 8.5 & 937.652 & 937.710 & -0.058 & 939.298 & 939.227 & -0.029 \\
\hline 9.5 & 946.960 & 947.003 & -0.043 & 948.654 & 948.679 & -0.025 \\
\hline 10.5 & 957.237 & 957.285 & -0.048 & 959.079 & 959.116 & -0.037 \\
\hline 11.5 & 968.496 & 968.556 & -0.060 & 970.510 & 970.539 & -0.029 \\
\hline 12.5 & 980.759 & 980.816 & -0.057 & 982.915 & 982.948 & -0.033 \\
\hline 13.5 & 994.001 & 994.065 & -0.064 & 996.306 & 996.342 & -0.036 \\
\hline 14.5 & 16008.248 & 16008.304 & -0.056 & 16010.692 & 16010.722 & -0.030 \\
\hline 15.5 & 023.490 & 023.533 & -0.043 & 026.058 & 026.088 & -0.030 \\
\hline 16.5 & 039.707 & 039.751 & -0.044 & 042.418 & 042.440 & -0.022 \\
\hline 17.5 & 056.916 & 056.960 & -0.044 & 059.756 & 059.778 & -0.022 \\
\hline 18.5 & 075.116 & 075.159 & -0.043 & 078.092 & 078.102 & -0.010 \\
\hline 19.5 & 094.332 & 094.348 & -0.016 & 097.401 & 097.413 & -0.012 \\
\hline 20.5 & 114.507 & 114.529 & -0.022 & 117.698 & 117.709 & -0.011 \\
\hline 21.5 & 135.687 & 135.700 & -0.013 & 138.985 & 138.992 & -0.007 \\
\hline 22.5 & 157.857 & 157.862 & -0.005 & 161.253 & 161.262 & -0.009 \\
\hline 23.5 & 181.000 & 181.015 & -0.015 & 184.514 & 184.519 & -0.005 \\
\hline 24.5 & 205.140 & 205.159 & -0.019 & 208.754 & 208.762 & -0.008 \\
\hline 25.5 & 230.290 & 230.295 & -0.005 & 234.000 & 233.992 & 0.008 \\
\hline 26.5 & 256.429 & 256.421 & 0.008 & 260.214 & 260.209 & 0.005 \\
\hline 27.5 & 283.549 & 283.539 & 0.010 & 287.421 & 287.414 & 0.007 \\
\hline 28.5 & 311.657 & 311.649 & 0.008 & 315.613 & 315.605 & 0.008 \\
\hline 29.5 & 340.768 & 340.749 & 0.019 & 344.790 & 344.784 & 0.006 \\
\hline 30.5 & 370.864 & 370.841 & 0.023 & 374.967 & 374.950 & 0.017 \\
\hline 31.5 & 401.933 & 401.924 & 0.009 & 406.121 & 406.103 & 0.018 \\
\hline 32.5 & 434.028 & 433.999 & 0.029 & 438.264 & 438.243 & 0.021 \\
\hline 33.5 & 467.097 & 467.064 & 0.033 & 471.407 & 471.371 & 0.036 \\
\hline 34.5 & 501.160 & 501.120 & 0.040 & 505.510 & 505.486 & 0.024 \\
\hline 35.5 & 536.217 & 536.167 & 0.050 & 540.631 & 540.598 & 0.043 \\
\hline 36.5 & 572.241 & 572.205 & 0.036 & 576.701 & 576.678 & 0.023 \\
\hline 37.5 & 609.304 & 609.234 & 0.070 & 613.791 & 613.755 & 0.036 \\
\hline 38.5 & 647.324 & 647.253 & 0.071 & 651.867 & 651.818 & 0.049 \\
\hline 39.5 & 686.347 & 686.261 & 0.086 & 690.900 & 690.869 & 0.031 \\
\hline 40.5 & $726 \cdot 336$ & 726.260 & 0.076 & 730.946 & 730.906 & 0.040 \\
\hline 41.5 & 767.293 & 767.249 & 0.044 & 771.964 & 771.930 & 0.034 \\
\hline 12.5 & 809.306 & 809.227 & 0.079 & 813.980 & 813.941 & 0.039 \\
\hline 43.5 & $852.26 \theta$ & 852.194 & 0.074 & 856.996 & 856.937 & 0.059 \\
\hline 44.5 & 896.229 & 896.150 & 0.079 & 900.965 & 900.920 & 0.045 \\
\hline 45.5 & 941.171 & 941.094 & 0.077 & 945.919 & 945.889 & 0.030 \\
\hline 46.5 & 987.048 & 987.027 & 0.021 & 991.869 & 991.843 & 0.026 \\
\hline
\end{tabular}
levels of the ${ }^{4} \Sigma^{-}$state are then simply the eigenvalues, $F_{\Omega, e}^{\prime \prime}(J)$ and $F_{\Omega, f}^{\prime \prime}(J)$, of the

TABLE IXa

"Experimental" and Calculated ${ }^{4} \Pi_{-1 / 2}$ Rotational Energy Levels $\left(\mathrm{cm}^{-1}\right)$ 
${ }^{4} \Sigma^{-}$matrices. Thus, Eqs. (12) and (13) yield the "experimental" rotational energy levels, $T_{\Omega, e}^{r}(J)$ and $T_{\Omega, f}^{\prime}(J)$, of the ${ }^{4} \Pi$ state by adding the appropriate measured line frequencies to $F_{\Omega, e}^{\prime \prime}(J)$ and $F_{\Omega, f}^{\prime \prime}(J)$. The calculated ${ }^{4} \Pi$ rotational levels are obtained from the values of $T_{\Omega}$ (Table VIII) and the eigenvalues, $F_{\Omega, e}^{\prime}(J)$ and $F_{\Omega, f}^{\prime}(J)$, of the ${ }^{4} \Pi$ matrices. These calculated and "experimental" ${ }^{4} \Pi$ rotational levels and their differences are given in Table IX for each ${ }^{4} \Pi_{\Omega}$ substate.

The calculated lambda doubling for each substate, $\Delta v_{\Omega}^{r A I C}(J)$ is, by definition, equal to $F_{\Omega, e}^{\prime}(J)-F_{\Omega, f}^{\prime}(J)$. The "experimental" lambda doubling $T_{\Omega, e}^{\prime}(J)-T_{\Omega, f}^{\prime}(J)$. Both lambda doublings are given in Table X. From Table $\mathrm{X}$ we see at once that the calculated lambda doubling of the ${ }^{4} \Pi_{3 / 2}$ substate is much smaller than the observed. On the other hand, $H_{\Lambda}$ predicts the lambda doubling of the other three substates quite satisfactorily. The two tables show clearly where the calculated and "experimental" ${ }^{4} \Pi$ levels differ.

The results given here indicate that a much more satisfactory theoretical formula for $H_{\Delta}$ is needed to adequately describe the lambda doubling of the ${ }^{4} \Pi_{3 / 2}$ substate.

TABLE IXb

"Experimental" and Calculated ${ }^{4} \Pi_{1 / 2}$ Rotational Energy Levels $\left(\mathrm{cm}^{-1}\right)$

\begin{tabular}{|c|c|c|c|c|c|c|}
\hline \multirow[b]{2}{*}{$J$} & \multicolumn{3}{|c|}{ E LEVELS } & \multicolumn{3}{|c|}{ F LEVELS } \\
\hline & EXPTL . & CALCD. & DTFE. & EXPTL. & CALCD. & DIFE. \\
\hline 3.5 & 15973.838 & 15973.793 & 0.045 & 15972.916 & 15972.899 & 0.017 \\
\hline $4+5$ & 978.530 & 978.498 & 0.032 & 977.402 & 977.384 & 0.018 \\
\hline 5.5 & 984.277 & 984.223 & 0.054 & 982.879 & 982.890 & -0.011 \\
\hline 6.5 & 991.021 & 990.967 & 0.054 & 989.405 & 989.417 & -0.012 \\
\hline 7.5 & 998.766 & 998.731 & 0.035 & 996.946 & 996.966 & -0.020 \\
\hline 8.5 & 16007.561 & 16007.513 & $0.04 \mathrm{~B}$ & 16005.509 & 16005.536 & -0.027 \\
\hline 9.5 & 017.354 & 017.314 & 0.040 & 025.082 & 015.127 & -0.045 \\
\hline 20.5 & $028+168$ & 028.132 & 0.036 & 025.702 & 025.739 & -0.037 \\
\hline 11.5 & 039.399 & 039.968 & 0.031 & 037.315 & 037.371 & -0.056 \\
\hline 12.5 & 052.845 & 052.820 & 0.025 & 049.965 & 050.024 & -0.059 \\
\hline 13.5 & 066.702 & 066.689 & 0.013 & 063.623 & 063.696 & -0.073 \\
\hline 14.5 & 081.581 & 081.574 & 0.007 & 078.302 & 078.389 & -0.087 \\
\hline 1.5 .5 & 097.476 & 097.474 & 0.002 & 094.017 & 094.100 & -0.083 \\
\hline 16.5 & 114.382 & 114.388 & -0.006 & 110.742 & 110.830 & -0.088 \\
\hline 17.5 & 132.299 & 132.317 & -0.018 & 128.489 & 128.578 & -0.089 \\
\hline 1.8 .5 & 151.231 & 151.259 & -0.028 & 147.245 & 147.344 & -0.099 \\
\hline 29.5 & 171.184 & 171.214 & -0.030 & 167.036 & 167.128 & -0.092 \\
\hline 70.5 & 192.143 & 192.181 & -0.038 & 187.832 & 187.928 & -0.096 \\
\hline 21.5 & $214=118$ & 214.160 & -0.042 & 209.635 & 209.744 & -0.109 \\
\hline 22.5 & 237.103 & 237.151 & -0.048 & 232.477 & 232.575 & -0.098 \\
\hline 23.5 & 261.099 & 261.151 & -0.052 & 256.329 & 256.421 & -0.092 \\
\hline 24.5 & 286.104 & 286.162 & -0.058 & 281.183 & 281.282 & -0.099 \\
\hline 25.5 & 312.124 & 312.181 & -0.057 & 307.056 & 307.156 & -0.100 \\
\hline 26.5 & 339.152 & 339.210 & -0.058 & 333.936 & 334.043 & -0.107 \\
\hline 27.5 & 367.182 & 367.246 & -0.064 & 361.854 & 361.942 & -0.088 \\
\hline 28.5 & 396.228 & 396.290 & -0.062 & 390.780 & 390.852 & -0.072 \\
\hline 29.5 & 426.278 & 426.340 & -0.062 & 420.696 & 420.774 & -0.078 \\
\hline 30.5 & 457.336 & 457.396 & -0.060 & 451.638 & 451.705 & -0.067 \\
\hline 31.5 & 489.400 & 489.458 & -0.058 & 483.574 & 483.646 & -0.072 \\
\hline 32.5 & 6522.476 & 522.525 & -0.049 & 516.543 & 516.596 & -0.053 \\
\hline 33.5 & 556,547 & 556.595 & -0.048 & 550.510 & 550.553 & -0.043 \\
\hline 34.5 & 591.631 & 591.670 & -0.039 & 585.495 & 585.518 & -0.023 \\
\hline 35.5 & 627.700 & 627.746 & -0.046 & 621.475 & 621.489 & -0.014 \\
\hline 36.5 & 664.790 & 664.825 & -0.035 & 659.473 & 658.465 & 0.008 \\
\hline 37.5 & 702.877 & 702.906 & -0.029 & 696.461 & 696.447 & 0.014 \\
\hline 38.5 & $741=964$ & 741.987 & -0.023 & $735 \times 462$ & 735.433 & 0.029 \\
\hline 39.5 & 782.061 & 782.069 & -0.008 & 775.461 & 775.422 & 0.079 \\
\hline 40.5 & 823,145 & 923.150 & -0.005 & 816.463 & 816.414 & 0.049 \\
\hline 41.5 & 865.225 & 865.229 & -0.004 & 858.474 & 858.407 & 0.067 \\
\hline 42.5 & 908.323 & 908.307 & 0.016 & 901.485 & 901.402 & 0.083 \\
\hline 43.5 & 952.415 & 952.382 & 0.033 & 945.478 & 945.397 & 0.081 \\
\hline
\end{tabular}


TABLE IXc

"Experimental" and Calculated ${ }^{4} \Pi_{3 / 2}$ Rotational Energy Levels $\left(\mathrm{cm}^{-1}\right)$

\begin{tabular}{|c|c|c|c|c|c|c|}
\hline \multirow[b]{2}{*}{$\mathrm{J}$} & \multicolumn{3}{|c|}{ E LEVELS } & \multicolumn{3}{|c|}{ F LEVELS } \\
\hline & EXPTL. & CALCD. & DIFF. & EXPTL. & CALCD. & DIFF. \\
\hline 3.5 & 16291.781 & 16291.596 & 0.185 & 16291.773 & 16291.596 & 0.177 \\
\hline 4.5 & 296.319 & 296.142 & 0.177 & 296.318 & 296.142 & 0.176 \\
\hline 5.5 & 301.872 & 301.699 & 0.173 & 301.877 & 301.699 & 0.178 \\
\hline 6.5 & 308.438 & 308.265 & 0.173 & 308.440 & 308.265 & 0.175 \\
\hline 7.5 & 316.013 & 315.842 & 0.171 & 326.013 & 315.842 & 0.171 \\
\hline 8.5 & 324.599 & 324.428 & 0.171 & 324.591 & 324.428 & 0.163 \\
\hline 9.5 & 334.195 & 334.025 & 0.170 & 334.183 & 334.024 & 0.159 \\
\hline 10.5 & 344.801 & 344.630 & 0.171 & 344.794 & 344.630 & 0.164 \\
\hline 11.5 & 356.409 & 356.246 & 0.163 & 356.403 & 356.246 & 0.157 \\
\hline 12.5 & 369.034 & 368.871 & 0.163 & 369.028 & 368.871 & 0.157 \\
\hline 13.5 & 382.667 & 382.506 & 0.161 & 382.660 & 382.505 & 0.155 \\
\hline 14.5 & 397.307 & 397.149 & 0.158 & 397.303 & 397.149 & 0.154 \\
\hline 15.5 & 412.953 & 412.802 & 0.151 & 412.951 & 412.801 & 0.150 \\
\hline 16.5 & 429.609 & 429.463 & 0.146 & 429.594 & 429.462 & 0.132 \\
\hline 17.5 & 447.282 & 447.133 & 0.149 & 447.266 & 447.132 & 0.134 \\
\hline $\begin{array}{l}18.5 \\
19.5\end{array}$ & $\begin{array}{r}465.959 \\
485.636\end{array}$ & $\begin{array}{l}465.811 \\
485.498\end{array}$ & $\begin{array}{l}0.148 \\
0.138\end{array}$ & $\begin{array}{l}465.953 \\
485.620\end{array}$ & $\begin{array}{l}465.811 \\
485.497\end{array}$ & $\begin{array}{l}0.142 \\
0.123\end{array}$ \\
\hline 20.5 & 506.330 & 506.193 & 0.137 & 506.311 & 506.191 & 0.120 \\
\hline 21.5 & 528.030 & 527.895 & 0.135 & 527.998 & 527.894 & 0.104 \\
\hline 22.5 & 550.735 & 550.605 & 0.130 & 550.710 & 550.603 & 0.107 \\
\hline 23.5 & 574.449 & 574.322 & 0.127 & 574.423 & 574.320 & 0.103 \\
\hline 24.5 & 599,166 & 599.045 & 0.121 & 599.126 & 599.044 & 0.082 \\
\hline 25.5 & 624.890 & 624.776 & 0.114 & 624.860 & 624.774 & 0.086 \\
\hline 26.5 & 651.624 & 651.513 & 0.111 & 651.587 & 651.511 & 0.076 \\
\hline 27.5 & 679.365 & 679.256 & 0.109 & 679.324 & 679.253 & 0.071 \\
\hline 28.5 & 708.106 & 708.005 & 0.101 & 708.032 & 708.002 & 0.030 \\
\hline 29.5 & 737.851 & 737.759 & 0.092 & 737.811 & 737.756 & 0.055 \\
\hline 30.5 & 768.609 & 768.518 & 0.091 & 768.554 & 768.514 & 0.040 \\
\hline 31.5 & 800.358 & 800.282 & 0.076 & 800.309 & $800+278$ & 0.031 \\
\hline 32.5 & 833.126 & 833.050 & 0.076 & 833.064 & 833.046 & 0.018 \\
\hline 33.5 & 866.889 & 866.822 & 0.067 & 866.816 & 866.817 & -0.001 \\
\hline 34.5 & 901.656 & 901.598 & 0.058 & 901.586 & 901.593 & -0.007 \\
\hline 35.5 & 937.427 & 937.376 & 0.051 & 937.357 & 937.371 & -0.014 \\
\hline 36.5 & 974.199 & 974.158 & 0.041 & 974.118 & 974.152 & -0.034 \\
\hline 37.5 & 17011.976 & 17011.942 & 0.034 & 17011.885 & 17011.935 & -0.050 \\
\hline 38.5 & 050.750 & 050.727 & 0.023 & 050.653 & 050.721 & -0.068 \\
\hline 39.5 & 090.527 & 090.514 & 0.013 & 090.426 & 090.507 & -0.081 \\
\hline 40.5 & 131.300 & 131.302 & -0.002 & 131.191 & 131.295 & -0.104 \\
\hline 41.5 & 173.079 & 173.091 & -0.012 & 172.949 & 173.082 & -0.133 \\
\hline 42.5 & 215.856 & 215.879 & -0.023 & 215.734 & 215.870 & -0.136 \\
\hline 43.5 & 259.630 & 259.667 & -0.037 & 259.503 & 259.658 & -0.155 \\
\hline 44.5 & 304.406 & 304.454 & -0.048 & 304.268 & 304.444 & -0.176 \\
\hline 45.5 & 350.178 & 350.240 & -0.062 & 350.031 & 350.229 & -0.198 \\
\hline 46.5 & 396.941 & 397.023 & -0.082 & 396.790 & 397.011 & -0.221 \\
\hline 47.5 & 444.710 & 444.803 & -0.093 & 444.550 & 444.791 & -0.241 \\
\hline 48.5 & 493.472 & 493.581 & -0.109 & 493.299 & 493.568 & -0.269 \\
\hline 49.5 & 543.228 & 543.355 & -0.127 & 543.052 & 543.341 & -0.289 \\
\hline 50.5 & 593.981 & 594.124 & -0.143 & 593.794 & 594.110 & -0.316 \\
\hline 51.5 & 645.738 & 645.889 & -0.151 & 645.524 & 645.873 & -0.349 \\
\hline 52.5 & 698.464 & 698.648 & -0.184 & 698.262 & 698.631 & -0.369 \\
\hline 53.5 & 752.196 & 752.400 & -0.204 & 751.986 & 752.383 & -0.397 \\
\hline 54.5 & 806.921 & 807.146 & -0.225 & 806.687 & 807.128 & -0.441 \\
\hline 55.5 & 862.642 & 862.885 & -0.243 & 862.372 & 862.866 & -0.494 \\
\hline 56.5 & 919.354 & 919.616 & -0.262 & 919.083 & 919.596 & -0.513 \\
\hline
\end{tabular}

The way in which the strong central features (Fig. $2 b$ in particular) depend very subtly upon this has already been noted. Changing $H_{\mathrm{A}}$ will produce changes in the values of some of the diagonal and off-diagonal elements in Table II. Corrections will then need to be made to the $T_{\Omega}$ in order to improve the fit still further and consequently improve the values of the ${ }^{4} \Pi$ molecular constants. This work is now being undertaken. 
TABLE IXd

"Experimental" and Calculated ${ }^{4} \Pi_{3 / 2}$ Rotational Energy Levels $\left(\mathrm{cm}^{-1}\right)$

\begin{tabular}{|c|c|c|c|c|c|c|}
\hline \multirow[b]{2}{*}{$\mathfrak{J}$} & \multicolumn{3}{|c|}{ E LEVELS } & \multicolumn{3}{|c|}{ F LEVELS } \\
\hline & EXPTL. & CALCD. & DIFF. & EXPTL. & CALCD. & DIFF. \\
\hline $7+5$ & 16747.791 & 16747.872 & -0.081 & 16747.794 & 16747.872 & -0.078 \\
\hline 8.5 & 756.386 & 756.470 & -0.084 & 756.388 & 756.470 & $-0,082$ \\
\hline 9.5 & 766.000 & 766.060 & -0.080 & 766.008 & 765.080 & -0.072 \\
\hline 10.5 & 776.521 & 776.701 & -0.080 & 776.631 & 776.701 & -0.070 \\
\hline 11.5 & 788.254 & 788.333 & -0.079 & 788.261 & 788.333 & -0.072 \\
\hline 12.5 & 800.898 & 800.976 & -0.078 & 800.907 & 800.976 & -0.069 \\
\hline 13.5 & 814.556 & 814.629 & -0.073 & $814 \times 555$ & 814.629 & -0.074 \\
\hline 14.5 & 829.227 & 829.293 & -0.066 & 829.221 & 829.293 & -0.072 \\
\hline 15.5 & 844.896 & 844.968 & -0.072 & 844.897 & 844.968 & -0.071 \\
\hline 16.5 & 861.576 & 861.653 & -0.077 & 861.581 & 851.653 & -0.072 \\
\hline 17.5 & 879.278 & 879.348 & -0.070 & 879.281 & 879.348 & -0.067 \\
\hline 18.5 & 897.976 & 898.052 & -0.076 & 897.976 & 898.052 & -0.076 \\
\hline 19.5 & 917.703 & 917.766 & -0.063 & 917.704 & 917.766 & -0.062 \\
\hline 20.5 & 938.421 & 938.490 & -0.069 & 938.421 & 938.490 & -0.069 \\
\hline 21.5 & 960.160 & 960.223 & -0.063 & 960.162 & 960.223 & -0.061 \\
\hline 22.5 & 982.912 & 982.964 & -0.052 & 982.901 & 982.964 & -0.063 \\
\hline 23.5 & 17006.661 & 17006.714 & -0.053 & 17006.657 & 17006.715 & -0.058 \\
\hline 24.5 & 031.427 & 031.473 & -0.046 & 031.419 & 031.473 & -0.054 \\
\hline 25.5 & 057.194 & 057.240 & -0.046 & 057.183 & 057.240 & -0.057 \\
\hline 26.5 & 083.970 & 084.014 & -0.044 & 083.962 & 084.014 & -0.052 \\
\hline 27.5 & 111.720 & 111.796 & -0.076 & 111.754 & 111.796 & -0.042 \\
\hline 28.5 & 140.545 & 140.585 & -0.040 & 140.542 & 140.585 & -0.043 \\
\hline 29.5 & 170.340 & 170.381 & -0.041 & 170.344 & 170.381 & -0.037 \\
\hline 30.5 & 201.150 & 201.183 & -0.033 & 201.147 & 201.183 & -0.036 \\
\hline 31.5 & 232.969 & 232.991 & -0.022 & 232.953 & 232.991 & -0.038 \\
\hline 32.5 & 265.781 & 265.805 & -0.024 & 265.779 & 265.805 & -0.026 \\
\hline 33.5 & 299.604 & 299.624 & -0.020 & 299.602 & 299.624 & -0.022 \\
\hline 34.5 & 334.434 & 334.449 & -0.01 .5 & 334.435 & 334.449 & -0.014 \\
\hline 35.5 & 370.256 & 370.277 & -0.021 & 370.270 & 370.277 & -0.007 \\
\hline 36.5 & 407.098 & 407.110 & -0.012 & 407.103 & 407.110 & -0.007 \\
\hline 37.5 & 444.944 & 444.947 & -0.003 & 444.947 & 444.947 & 0.000 \\
\hline 38.5 & 483.795 & 483.787 & 0.008 & 483.791 & 483.787 & 0.004 \\
\hline 39.5 & 523.639 & 523.629 & 0.010 & 523.639 & 523.629 & 0.010 \\
\hline 40.5 & 564.486 & 564.474 & 0.012 & 564.491 & 564.474 & 0.017 \\
\hline 41.5 & 606.342 & 606.321 & 0.021 & 606.340 & 606.321 & 0.019 \\
\hline 42.5 & 649.200 & 649.169 & 0.031 & 649.202 & 649.169 & 0.033 \\
\hline 43.5 & 693.054 & 693.018 & 0.036 & 693.049 & 693.018 & 0.031 \\
\hline 44.5 & 737.902 & 737.867 & 0.035 & 737.915 & 737.868 & 0.047 \\
\hline 45.5 & 783.773 & 783.737 & 0.056 & 783.771 & 783.717 & 0.054 \\
\hline 46.5 & 830.634 & 830.565 & 0.069 & 830.528 & 830.566 & 0.062 \\
\hline 47.5 & 879.465 & 878.412 & 0.053 & 878.478 & 878.413 & 0.065 \\
\hline 48.5 & 927.325 & 927.258 & 0.067 & 927.324 & 927.258 & 0.066 \\
\hline 49.5 & 977.182 & 977.101 & 0.081 & 977.178 & 977.101 & 0.077 \\
\hline 50.5 & 28028.035 & 18027.941 & 0.094 & 18028.033 & 18027.941 & 0.092 \\
\hline
\end{tabular}

\section{CONCLUSIONS}

The red system of MoN is undoubtedly a ${ }^{4} \Pi(a) \rightarrow{ }^{4} \Sigma^{-}(a)$ transition despite some problems in the agreement between the experimental and theoretical energy levels. The most surprising aspect of the ${ }^{4} \mathrm{II}$ state is the significant displacement of the $\Omega=-1 / 2$ component from the position expected for reasonable case (a) behavior. Even allowing for spin uncoupling, the level is shifted $\sim 175 \mathrm{~cm}^{-1}$ from its "expected" position and yet there is no evidence of rotational perturbations in this, or in any other, component. The inability of the present theoretical framework to correctly account for the $\Lambda$ doubling of the $\Omega=3 / 2$ component, together with the shift of the $\Omega=-1 / 2$ component, suggests either-or both-of a perturbation by a non- ${ }^{4} \Sigma$ state $\left({ }^{2} \Pi\right.$ ?) or the effects of uncoupling toward case d. Despite the inherent plausibility of this latter, since the $p \pi$ electron has much of a Rydberg character 
TABLE $X$

"Experimental" and Calculated Lambda-Doubling of the ${ }^{4} \Pi$ State $\left(\mathrm{cm}^{-1}\right)$

\begin{tabular}{|c|c|c|c|c|c|}
\hline \multirow[b]{2}{*}{$\mathbf{J}$} & \multicolumn{2}{|c|}{${ }^{4} \pi_{-1 / 2}$} & \multirow[b]{2}{*}{$\mathrm{J}$} & \multicolumn{2}{|c|}{${ }^{4} n 1 / 2$} \\
\hline & EXPTL. & CALCD. & & EXPTL. & CALCD. \\
\hline 2.5 & -0.522 & -0.518 & 3.5 & 0.922 & 0.893 \\
\hline 3.5 & -0.695 & -0.689 & 4.5 & 1.128 & 1.114 \\
\hline 4.5 & -0.843 & -0.858 & 5.5 & 1.398 & 1.333 \\
\hline 6.5 & -1.209 & -1.192 & 7.5 & 1.820 & 1.765 \\
\hline 5.5 & -1.045 & -1.026 & 6.5 & 1.616 & 1.550 \\
\hline 7.5 & -1.379 & -1.356 & B. 5 & 2.052 & 1.978 \\
\hline $\begin{array}{l}8.5 \\
9.5\end{array}$ & $\begin{array}{l}-1.546 \\
-1.694\end{array}$ & $\begin{array}{l}-1.517 \\
-1.676\end{array}$ & $\begin{array}{r}9.5 \\
10.5\end{array}$ & $\begin{array}{l}2.272 \\
2.466\end{array}$ & $\begin{array}{l}2.187 \\
2.394\end{array}$ \\
\hline 10.5 & -1.842 & -1.831 & 11.5 & 2.684 & 2.597 \\
\hline 11.5 & -2.014 & -1.983 & 12.5 & 2.880 & 2.797 \\
\hline 12.5 & -2.156 & -2.132 & 13.5 & 3.079 & 2.993 \\
\hline 13.5 & -2.305 & -2.277 & 14.5 & 3.279 & 3.185 \\
\hline 14.5 & -2.444 & -2.418 & 15.5 & 3.459 & 3.374 \\
\hline 15.5 & -2.568 & -2.556 & 16.5 & 3.640 & 3.558 \\
\hline 16.5 & -2.711 & -2.689 & 17.5 & 3.810 & 3.739 \\
\hline 17.5 & -2.840 & -2.818 & 18.5 & 3.986 & 3.915 \\
\hline 18.5 & -2.976 & -2.943 & 19.5 & 4.148 & 4.086 \\
\hline 19.5 & -3.069 & -3.064 & 20.5 & 4.311 & 4.254 \\
\hline 20.5 & -3.191 & -3.181 & 21.5 & 4.483 & 4.417 \\
\hline 21.5 & -3.298 & -3.293 & 22.5 & 4.626 & 4.576 \\
\hline 22.5 & -3.396 & -3.400 & 23.5 & 4.770 & 4.730 \\
\hline 23.5 & -3.514 & -3.504 & 24.5 & 4.921 & 4.880 \\
\hline 24.5 & -3.614 & -3.603 & 25.5 & 5.068 & 5.026 \\
\hline 25.5 & -3.710 & -3.698 & 26.5 & 5.216 & 5.167 \\
\hline 26.5 & -3.785 & -3.788 & 27.5 & 5.328 & 5.304 \\
\hline 27.5 & -3.872 & -3.874 & 28.5 & 5.448 & 5.437 \\
\hline 28.5 & -3.956 & -3.956 & 29.5 & 5.582 & 5.566 \\
\hline 29.5 & -4.022 & -4.034 & 30.5 & 5.698 & 5.691 \\
\hline 30.5 & -4.103 & -4.109 & 31.5 & 5.826 & 5.812 \\
\hline 31.5 & -4.188 & -4.179 & 32.5 & 5.933 & 5.929 \\
\hline 32.5 & -4.236 & -4.245 & 33.5 & 6.037 & 6.042 \\
\hline 33.5 & -4.310 & -4.307 & 34.5 & 6.136 & 6.152 \\
\hline 34.5 & -4.350 & -4.366 & 35.5 & 6.225 & 6.258 \\
\hline 35.5 & -4.414 & -4.421 & 36.5 & 6.317 & 6.360 \\
\hline 36.5 & -4.460 & -4.473 & 37.5 & 6.416 & 6.459 \\
\hline 37.5 & -4.487 & -4.521 & 38.5 & 6.502 & 6.554 \\
\hline 38.5 & -4.543 & -4.566 & 39.5 & 6.600 & 6.647 \\
\hline 39.5 & -4.553 & -4.607 & 40.5 & 6.682 & 6.736 \\
\hline 40.5 & -4.610 & -4.546 & 41.5 & 6.751 & 6.822 \\
\hline 41.5 & -4.671 & -4.681 & 42.5 & 6.838 & 6.905 \\
\hline 42.5 & -4.674 & -4.714 & 43.5 & 6.937 & 6.985 \\
\hline 43.5 & -4.728 & -4.743 & & & \\
\hline 44.5 & -4.736 & -4.770 & & & \\
\hline 45.5 & -4.748 & -4.794 & & & \\
\hline 46.5 & -4.821 & -4.816 & & & \\
\hline
\end{tabular}

(vide infra), preliminary calculations do not bear out this interpretation and the origin of the $\Lambda$ doubling and spin-orbit discrepancies remain unattributed.

The magnitude of the zero-field splitting of the ${ }^{4} \Sigma^{-}$state is extremely large and affords an excellent example of a "case (a)" ${ }^{4} \Sigma^{-}$state. This splitting indicates the importance of higher-order spin-orbit effects in the second-row transition elements and the importance of intermediate coupling for molecules containing these atoms.

The combination of the very small change in the upper- and lower-state $B$ values (of the order of $3.5 \%$ ), the magnitude of the spin-orbit coupling constant, and the characterization of the ground state as ${ }^{4} \Sigma^{-}$unambiguously assign this transition as highly localized upon the metal atom and of a pseudoligand-field type (31-33). Even though it is not correct to describe the molecule as $\mathrm{Mo}^{3+} \mathrm{N}^{3-}$, nonetheless the preponderant configuration for the ground term (34) is undoubtedly $s \sigma^{1} d \delta^{2}$. Similarly, it seems clear from the intensity of the transition that the excited state configuration is predominantly $d \delta^{2} p \pi^{1}$, where the $p \pi$ orbital is the Van Vleck "pure precession" 
TABLE X-Continued

\begin{tabular}{|c|c|c|c|c|c|}
\hline \multirow[b]{2}{*}{$J$} & \multicolumn{2}{|c|}{${ }^{4}{ }^{1 / 2}$} & \multirow[b]{2}{*}{3} & \multicolumn{2}{|c|}{$n_{5 / 2}$} \\
\hline & EXPTL . & CALCD. & & EXPTL. & CALCD. \\
\hline 3.5 & 0.008 & 0.000 & 7.5 & -0.003 & -0.000 \\
\hline 4.5 & 0.001 & 0.000 & 8.5 & -0.002 & -0.000 \\
\hline 5.5 & -0.005 & 0.000 & 9.5 & -0.000 & -0.000 \\
\hline 6.5 & -0.002 & 0.000 & 10.5 & -0.010 & $-0,000$ \\
\hline 7.5 & 0.000 & 0.000 & 11.5 & -0.007 & -0.000 \\
\hline a. 5 & 0.008 & 0.000 & 12.5 & -0.009 & -0.000 \\
\hline 9.5 & 0.012 & 0.000 & 13.5 & 0.002 & -0.000 \\
\hline 10.5 & 0.007 & 0.000 & 14.5 & 0.006 & -0.000 \\
\hline 11.5 & 0.006 & 0.000 & 15.5 & -0.001 & -0.000 \\
\hline 12.5 & 0.006 & 0.000 & 16.5 & -0.005 & -0.000 \\
\hline 13.5 & 0.007 & $0.0 \mathrm{nn}$ & 17.5 & -0.100 .3 & -1.000 \\
\hline 14.5 & 0.004 & 0.000 & 38.5 & 0.000 & -0.000 \\
\hline 15.5 & 0.002 & 0.000 & 39.5 & -0.001 & -0.000 \\
\hline 16.5 & 0.015 & 0.001 & 20.5 & 0.000 & -0.000 \\
\hline 17.5 & 0.016 & 0.001 & 21.5 & -0.002 & -0.000 \\
\hline 18.5 & 0.006 & 0.001 & 22.5 & 0.011 & -0.000 \\
\hline 19.5 & 0.016 & 0.001 & 23.5 & 0.004 & -0.000 \\
\hline 20.5 & 0.019 & 0.001 & 24.5 & 0.008 & -0.000 \\
\hline 21.5 & 0.032 & 0.001 & 25.5 & 0.011 & -0.000 \\
\hline 22.5 & 0.025 & 0.001 & 26.5 & 0.008 & -0.000 \\
\hline 23.5 & 0.026 & 0.002 & 27.5 & -0.004 & -0.000 \\
\hline 24.5 & 0.040 & 0.002 & 28.5 & 0.003 & -0.000 \\
\hline 25.5 & 0.030 & 0.002 & 29.5 & -0.004 & -0.000 \\
\hline 26.5 & 0.037 & 0.002 & 30.5 & 0.003 & -0.000 \\
\hline 27.5 & 0.041 & 0.003 & 31.5 & 0.015 & -0.000 \\
\hline 28.5 & 0.074 & 0.003 & 32.5 & 0.002 & -0.000 \\
\hline 29.5 & 0.040 & 0.003 & 33.5 & 0.002 & -0.000 \\
\hline 30.5 & 0.055 & 0.003 & 34.5 & -0.001 & -0.000 \\
\hline 31.5 & 0.049 & 0.004 & 35.5 & -0.014 & -0.000 \\
\hline 32.5 & 0.062 & 0.004 & 36.5 & -0.005 & -0.000 \\
\hline 33.5 & 0.073 & 0.005 & 37.5 & -0.003 & -0.000 \\
\hline 34.5 & 0.070 & 0.005 & 38.5 & 0.004 & -0.000 \\
\hline 35.5 & 0.070 & 0.005 & 39.5 & 0.000 & -0.000 \\
\hline 36.5 & 0.081 & 0.006 & 40.5 & -0.005 & -0.000 \\
\hline 37.5 & 0.091 & 0.006 & 41.5 & 0.002 & -0.000 \\
\hline 38.5 & 6.097 & 0.007 & $42 \times 5$ & -0.002 & $-0,000$ \\
\hline 39.5 & 0.101 & 0.007 & 43.5 & 0.005 & -0.000 \\
\hline 40.5 & 0.109 & 0.008 & 44.5 & -0.013 & -0.000 \\
\hline 41.5 & 0.130 & 0.009 & 45.5 & 0.002 & -0.000 \\
\hline 42.5 & 0.122 & 0.009 & 46.5 & 0.005 & -0.000 \\
\hline 43.5 & 0.127 & 0.010 & 47.5 & $-0,01.3$ & -0.000 \\
\hline 44.5 & 0.138 & 0.010 & 48.5 & $0.00 \mathrm{2}$ & -0.001 \\
\hline 45.5 & 0.147 & 0.011 & 49.5 & 0.004 & -0.001 \\
\hline 46.5 & 0.151 & 0.012 & 50.5 & 0.002 & -0.001 \\
\hline 4). & 0.160 & 0.012 & & & \\
\hline 48.5 & 0.173 & 0.013 & & & \\
\hline 49.5 & 0.1 .76 & 0.014 & & & \\
\hline 50.5 & 0.187 & 0.015 & & & \\
\hline 51.5 & 0.214 & 0.015 & & & \\
\hline 52.5 & $\begin{array}{l}0.202 \\
0.210\end{array}$ & $\begin{array}{l}0.016 \\
0.017\end{array}$ & & & \\
\hline $\begin{array}{l}53.5 \\
54.5\end{array}$ & $\begin{array}{l}0.210 \\
0.234\end{array}$ & $\begin{array}{l}0.017 \\
0.018\end{array}$ & & & \\
\hline $\begin{array}{l}54.5 \\
55.5\end{array}$ & $\begin{array}{l}0.234 \\
0.270\end{array}$ & $\begin{array}{l}0.018 \\
0.019\end{array}$ & & & \\
\hline 56.5 & 0.271 & 0.020 & & & \\
\hline
\end{tabular}

partner of a higher-lying $p \sigma(35)$, with both of these orbitals being, predominantly, the "Stark" components of the atomic $5 p$ orbital essentially localized on the Mo atom. In this regard, it is the $5 s \sigma \rightarrow 5 p \pi$ analog of the $A \rightarrow X$ system of TiN (1), the $A \rightarrow X$ system of $\mathrm{ZrN} \mathrm{(3),} \mathrm{the} A^{3} \Phi \rightarrow X^{3} \Delta$ system of $\mathrm{NbN}(4)$, a similar system in $\mathrm{TaN}(9)$, and all of the individual analogs of these transitions in the transition metal oxide species ( $\mathrm{ScO}$, $\mathrm{TiO}, \mathrm{VO}, \mathrm{ZrO}, \mathrm{NbO}$, etc.) $(31,36)$. A more systematic semiempirical approach to this series of assignments has been worked out (37) and will be published elsewhere.

The present MoN system has been seen $(13,14)$ in low temperatures in a matrix where, of course, only the ${ }^{4} \Sigma_{1 / 2}^{-}$component is populated. These spectra also suggest the presence of the one-electron analog $s \sigma \rightarrow p \sigma$ at higher energies, as is seen for many other species $(3,38)$ and there are, indeed, fragments of other systems to higher energies in the gas phase which have not yet been analyzed (39). 
Finally, it seemed appropriate to search for the particularly strong, line-like, feature at $6123 \AA$ in the spectra of M- and S-type stars. Despite a careful search, only one feature in the spectrum of $\beta$-Pegasi (40), an M-type star, was found at this wavelength. The feature was originally ascribed to $\mathrm{TiO}$, but more recent spectra of TiO do not show any strong features at this precise wavelength. It is also interesting to note that the feature is given a precision in location of only about $0.5 \AA$ compared with the $0.02 \AA$ for many other features in this spectrum-thereby suggesting that it is not the usual kind of well-defined bandhead or atomic line. In the case of the red system of titanium nitride (l), most of the major bandheads are obscured by the prominent calcium principal series transition, but in molybdenum nitride these do not coincide. The precision of the present observations should be more than adequate to enable the red system of MoN to be identified in future stellar investigations.

APPENDIX: COMBINATION RELATIONS USED FOR THE PRELIMINARY AND INTERMEDIATE ANALYSES

Reference to Fig. 3 shows the following relations for the ${ }^{4} \Sigma_{\Omega}^{-}$substates

$$
\begin{aligned}
& F_{f}^{\prime \prime}(J)-F_{e}^{\prime \prime}(J+1)=P_{e d}(J+1)-Q_{e f}(J)=Q_{j e}(J+1)-R_{f f}(J) \\
& F_{f}^{\prime \prime}(J+1)-F_{e}^{\prime \prime}(J)=R_{e d}(J)-Q_{e f}(J+1)=Q_{f e}(J)-P_{f f}(J+1),
\end{aligned}
$$

and, from these, the following combination relations for each ${ }^{4} \Sigma_{\Omega}^{-}$substate can be formed

$$
\begin{aligned}
& \Delta_{1}^{a} F^{\prime \prime}(J)=R_{e c}(J)-Q_{e f}(J+1)+Q_{f e}(J)-P_{f f}(J+1) \\
& \Delta_{1}^{b} F^{\prime \prime}(J)=P_{e e}(J+1)-Q_{e f}(J)+Q_{f e}(J+1)-R_{f f}(J) .
\end{aligned}
$$

Also by reference to Fig. 3 it can be seen that, for each ${ }^{4} \Pi_{\Omega}$ substate,

$$
\begin{aligned}
& F_{f}^{\prime}(J+1)-F_{e}^{\prime}(J)=Q_{f e}(J+1)-P_{e d}(J+1)=R_{f f}(J)-Q_{e f}(J) \\
& F_{e}^{\prime}(J+1)-F_{f}^{\prime}(J)=Q_{e f}(J+1)-P_{f f}(J+1)=R_{e c}(J)-Q_{f d}(J),
\end{aligned}
$$

and, accordingly,

$$
\begin{aligned}
& \Delta_{1}^{a} F^{\prime}(J)=Q_{e f}(J+1)-P_{f f}(J+1)+R_{e \ell}(J)-Q_{f e}(J) \\
& \Delta_{1}^{b} F^{\prime}(J)=Q_{f e}(J+1)-P_{e c}(J+1)+R_{f f}(J)-Q_{e f}(J) .
\end{aligned}
$$

\section{ACKNOWLEDGMENTS}

We gratefully acknowledge that this work was made possible by the award of Grant GP 16089 from the National Science Foundation to T.M.D. We also acknowledge valuable comments from Dr. R. W. Field, Dr. A. J. Merer, and Dr. S. P. Davis.

RECEIVED: May 25, 1984

\section{REFERENCES}

1. T. M. Dunn, L. K. Hanson, And K. A. Rubinson, Canad. J. Phys. 48, 1657-1663 (1970).

2. J. K. BATES, N. L. RANieri, AND T. M. DunN, Canda. J. Phys. 54, 915-916 (1976). 
3. J. K. BATES AND T. M. DUN, Canad. J. Phys. 54, 1216-1223 (1976).

4. T. M. DUNN AND K. M. RAO, Nature (London) 222, 266-267 (1969).

5. J.-L. Féménias, C. Athénour, and T. M. DunN, J. Chem. Phys. 63, 2861-2868 (1975).

6. J. C. Howard and J. G. Conway, J. Chem. Phys. 43, 3055-3057 (1965).

7. S. G. Krishnamurti and M. SWaminathan, Curr. Sci. (India) 23, 258-262 (1954).

8. M. MANDICH, J, K. BATES, AND T. M. DUNN, to be published.

9. J. K. BATES AND T. M. DUNN, to be published.

10. J. K. BATES AND T. M. DUNN, to he published.

11. J. K. Bates AND D. M. Gruen, J. Chem. Phys. 70, 4428-4429 (1979).

12. J. K. Bates AND D. M. GRuen, High Temp. Sci. 10, 27-43 (1978).

13. J. K. BATES AND D. M. GRUEN, J. Mol Spectrose. 78, 284-297 (1979).

14. L. B. KNIGHT AND J. STEAdMAN, J. Chem. Phys. 76, 3378-3384 (1982).

15. D. W. Green, W. Korfmacher, And D. M. Gruen, J. Chem. Phys. 58, 404-405 (1973).

16. R. W. Martin And A. J. Merer, Canad. J. Phys. 51, 125-143 (1973).

17. R. W. Martin and A. J. Merer, Canad. $J$. Phys. 51, 634-643 (1973).

18. D. L. Albritton, A. L. SCHMEltekopf, W. J. HARRop, R. N. ZARE, AND J. CZARNY, J. Mol. Spectrosc. 67, 157-184 (1977).

19. L. VeSETH, Phys. Scr. 12, 125-128 (1975),

20. R. N. Zare, A. L. SChMELteKopf, W. J. HaRRop, AND D. L. Albritron, J. Mol. Spectrosc. 46, $37-66(1973)$.

21. A. S-C. Cheung, R. C. Hansen, ANd A. J. Merer, J. Mol. Spectrosc, 91, 165-208 (1982).

22. A. S-C. Cheung, A. W. TAYlor, and A. J. Merer, J. Mol. Spectrosc. 92, 391-409 (1982).

23. D. Cossart, H. LAVENDY, AND J. M. RobBle, J. Mol. Spectrose. 99, 369-406 (1983).

24. G. HeRzBERG, "Spectra of Diatomic Molecules," 2nd ed., pp. 240-245, Van Nostrand, Princeton, New Jersey, 1950.

25. I. Kopp AND J. T. Hovgen, Canad. J. Phys, 45, 2581-2596 (1981).

26. J. H. VAN VLECK, Rev. Mod. Phys. 23, 213-227 (1951).

27. R. S. Muluiken AND A. Christy, Phys. Rev. 38, 87-119 (1931).

28. A. BUDO AND I. KovaCS, Phys. Z. 45, 122-126 (1944).

29. J. P. CHANDLER, "STEPT: A Family of Routines for Optimization and the Fitting of Data," Catalog \#307, Quantum Chemistry Program Exchange, Department of Chemistry, Indiana University. Bloomington, Indiana, 1975.

30. D. W. Marquard, J. Soc. Indust. Appl. Math. 11, 431-441 (1963).

31. T. M. DunN, in "Physical Chemistry-An Advanced Treatise" (H. Eyring, D. Henderson, and W. Jost, Eds.), Vol. V, pp. 205-286, Academic Press, New York, 1970.

32. T. M. DUNN, in "Proceedings. XIIIth Colloquium Spectrocopicum Internationale, P5821 Ottawa, 1967," pp. 363-369, Adam Hilger, London, 1967.

33. H. G. Powel, Proc. Phys. Soc 57, 32-45 (1945).

34. C. K. Jorgensen, "Modern Aspects of Ligand Field Theory," Part V, p. 513, American Elsevier, New York. 1971.

35. J. H. VAN VleCK, Phys. Rev. 33, 467-506 (1929).

36. C. J. Cheetham and R. F. Barrow, Adv. High Temp. Chem. 1, 7-4l (1967).

37. R. C. CARLSON AND T. M. DUNN, submitted for publication.

38. A. ADAMS, W. KLEMPERER, AND T. M. DUnN, Canad. J. Phys. 46, 2213-2220 (1968).

39. J. K. BATES, "Optical Emission Spectra of Diatomic Metal Nitrides," Ph.D. Dissertation, The University of Michigan, 1975.

40. D, N. Davis, Astrophys, $J$. 106, 28-75 (1947), 